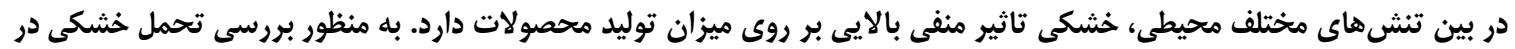

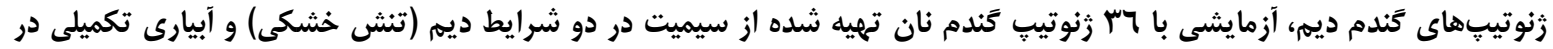

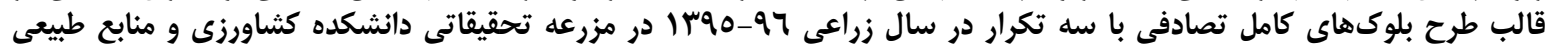

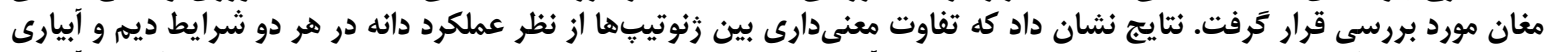

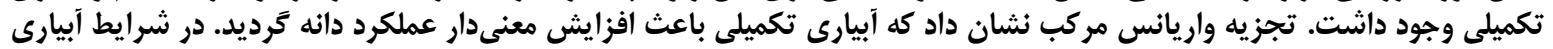

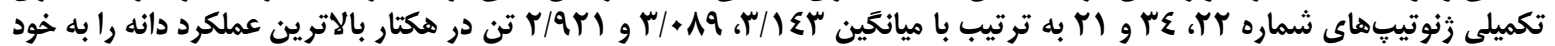

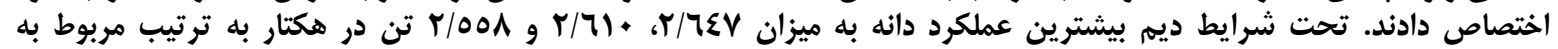

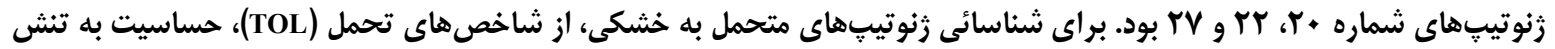

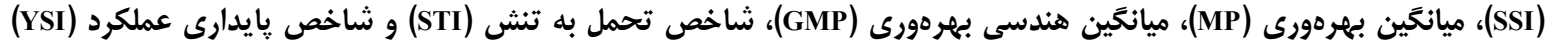

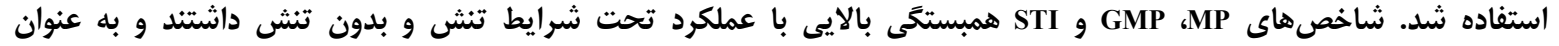

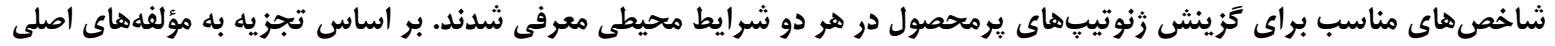

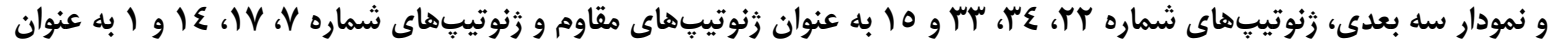

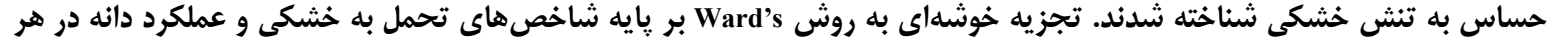

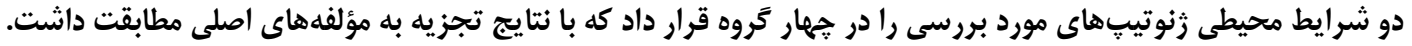

$$
\text { وازههاى كليدى: كَندم نان، آبيارى تكميلى، تنش خشكى، عملكرد دانه، شاخصهاى تحمل به تنش }
$$

بتوان با كمترين مقدار آب قابل دسترس، در طى مراحل حساس

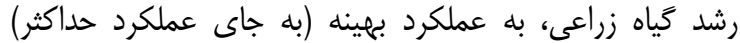

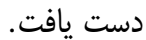
بلوم (أ) در مطالعات خود بيان كرد كه بيشترين كاهش

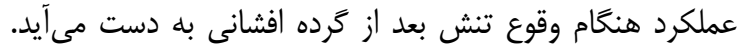

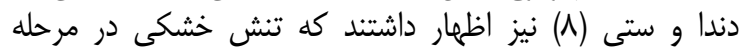

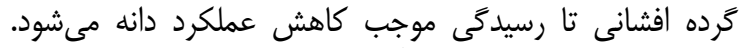

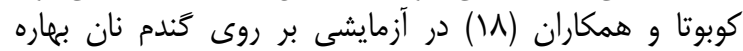

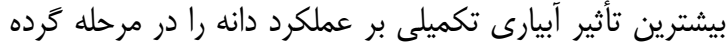
افشانى مشاهده نمودند.

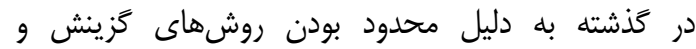

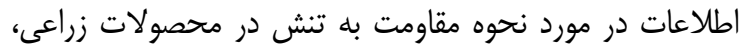

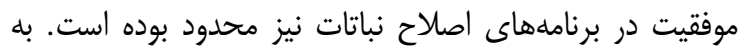

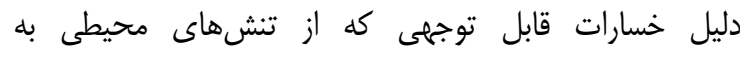

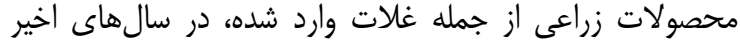

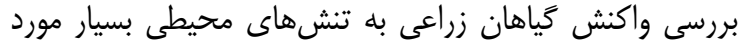

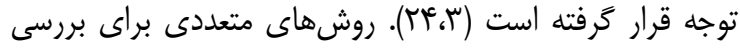

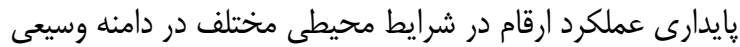

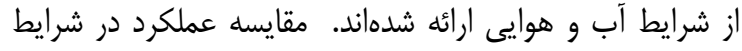

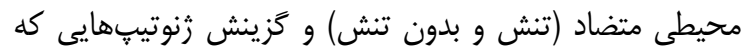

مقدمه تنشهاى محيطى را مىتوان به دو دسته عمده تنشهاى

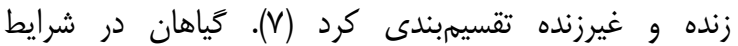
مزرعهاى در معرض تنشهاى غيرهن غيرنده متفاوتى مانند كمبود آب،

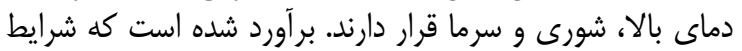

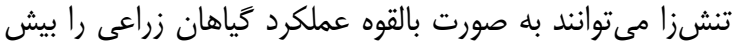

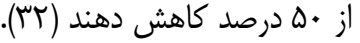

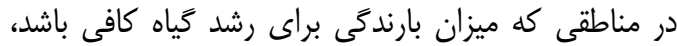

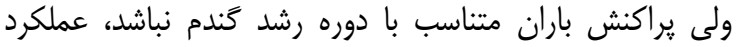

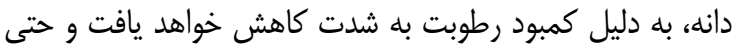

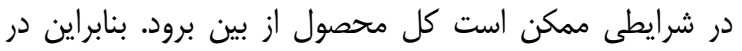

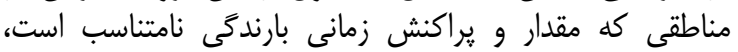

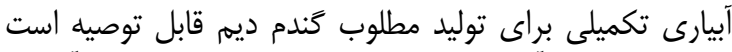

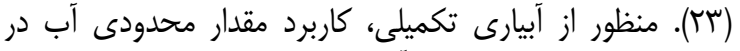

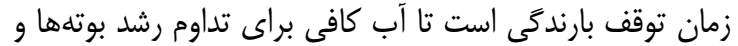

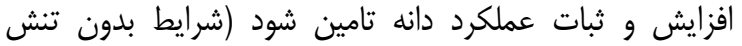

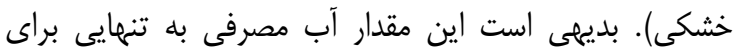

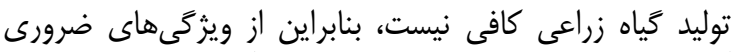

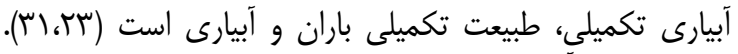

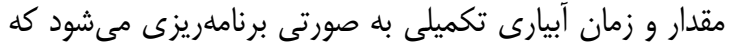


(STI)

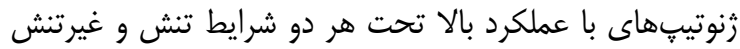

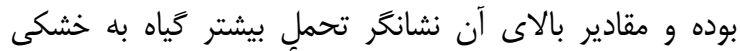

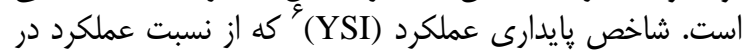

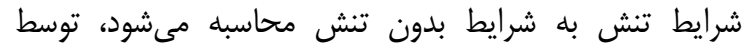

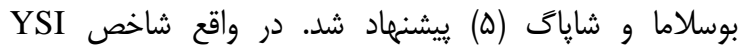

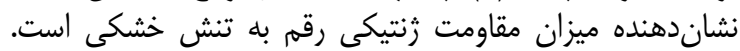

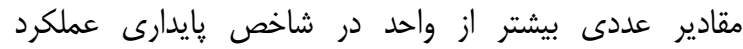
نشاندهنده حساسيت زنوتيٍ و ضعف پايدارى عملكرد در شرايط

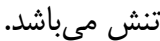

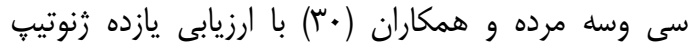

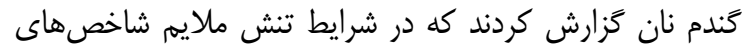
GMP و MP ، STI

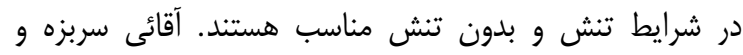

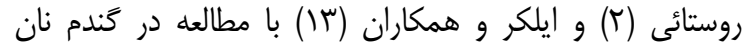

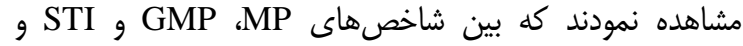

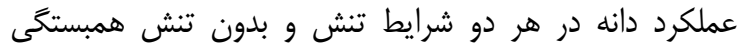

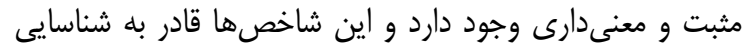

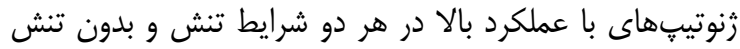

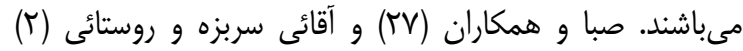

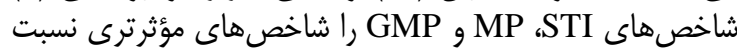

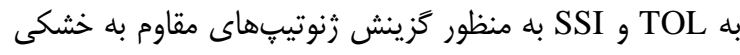
معرفى كردند.

هدف از اين تحقيق بررسى تاثير آبيارى تكميلى بر روى

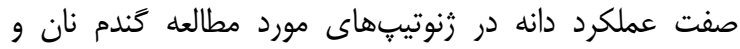

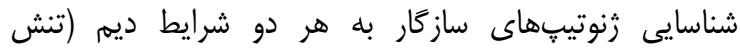

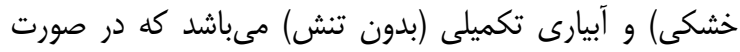

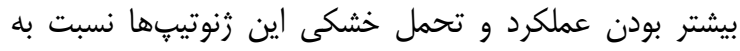

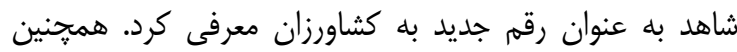

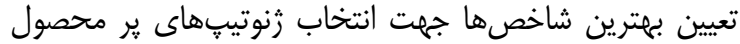
و متحمل به تنش خشكى مى باشد.

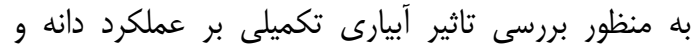

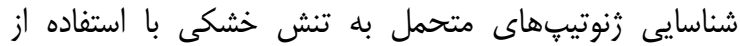

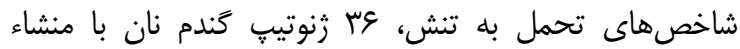

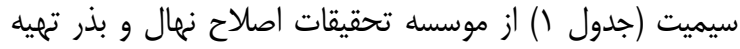

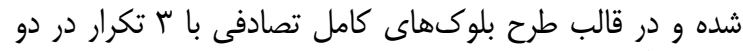

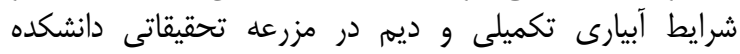

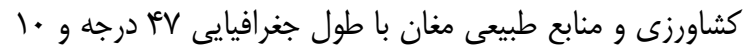

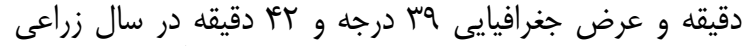

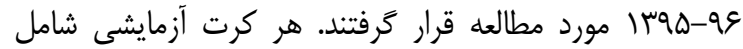

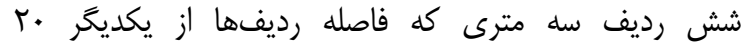

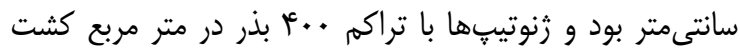

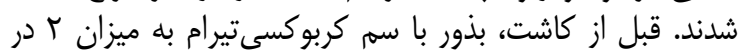

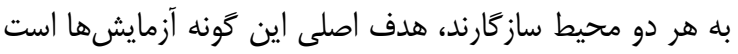

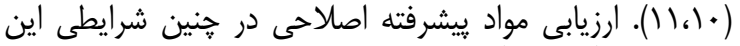

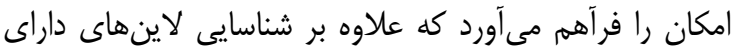

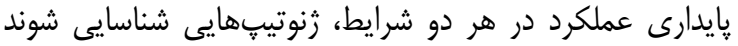

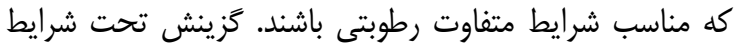

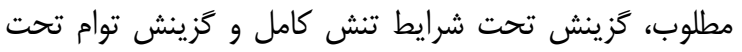

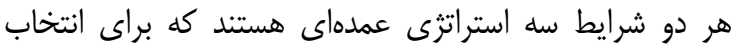

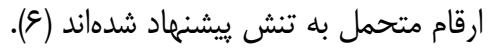

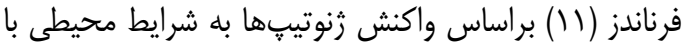

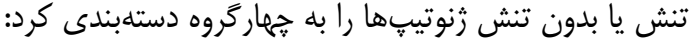

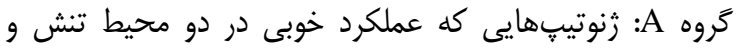

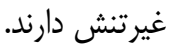
كَروه B: رنوتيڤهايى كه فقط عملكرد خوبى در محيط غيرتنش دارند.

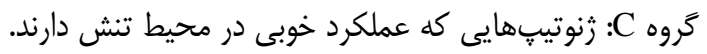

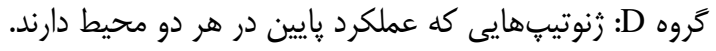

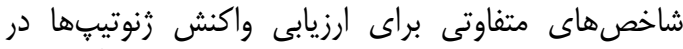
شرايط محيطى مختلف و تعيين مقاومت و و حساسيت آناسيت آنها ارائه

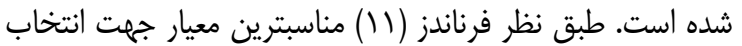

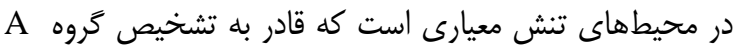

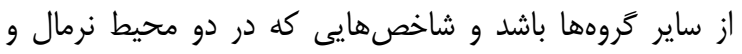

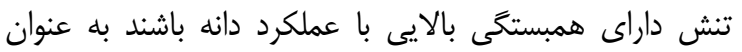

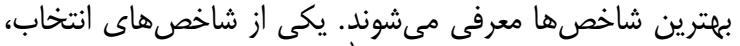

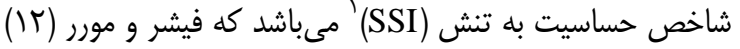

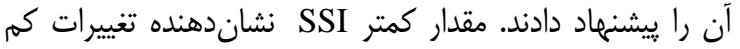

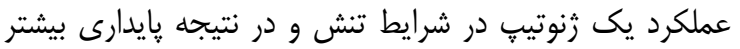

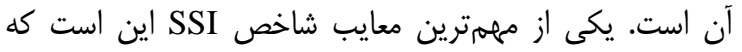

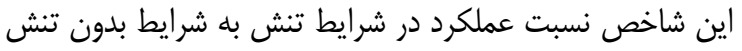

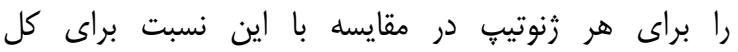

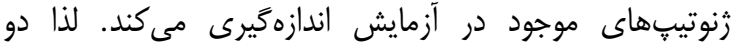

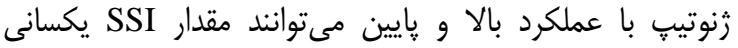

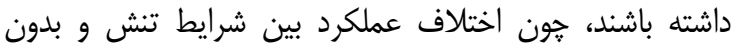

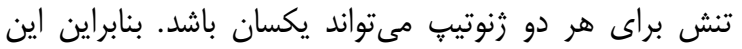

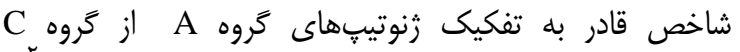

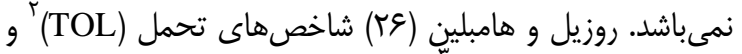

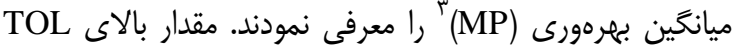

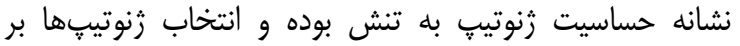

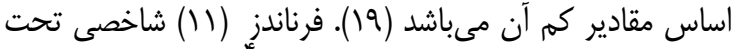

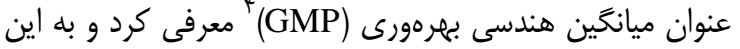

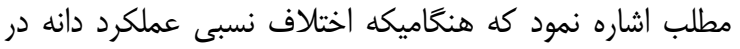

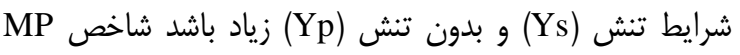

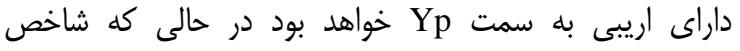

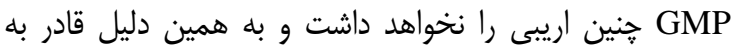

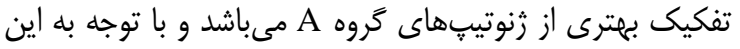
مسئله شاخص ديخرى تحت عنوان شاخص تحمل به به تنش 
همبستخى بين شاخصهاى تنش، تجزيه به به مؤلفههاى اصلى،

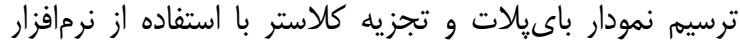

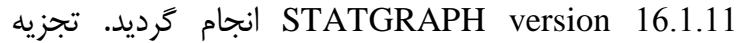
خوشهاى به روش Ward's بر مبناى مربع فاصله اقليدسى انجام

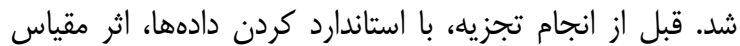

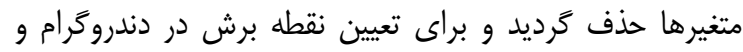

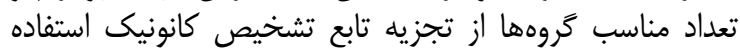

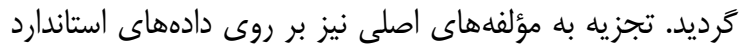

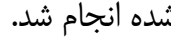

\section{تنايج و بحث تجزيه واريانس}

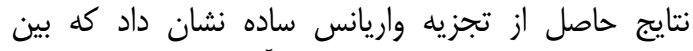

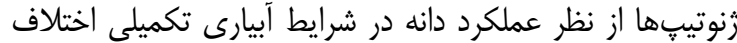

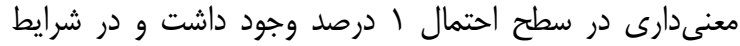

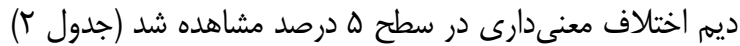

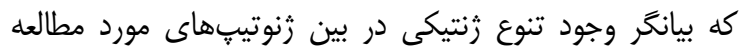

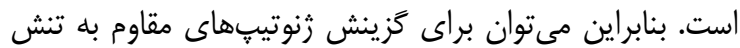

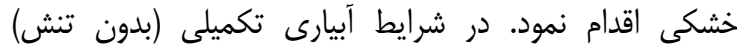

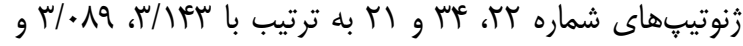

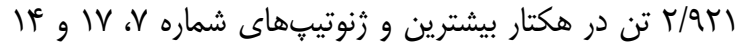

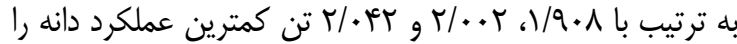

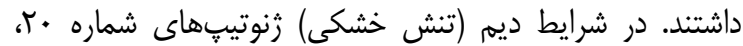

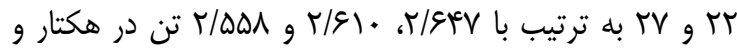

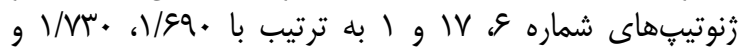

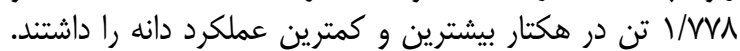

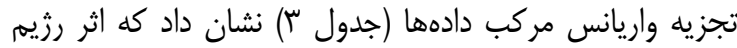

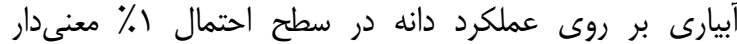

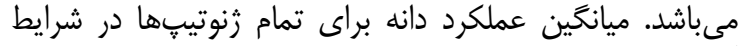

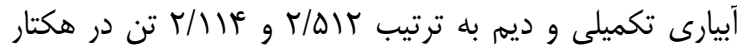

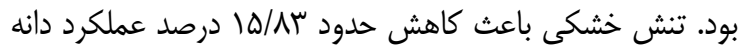

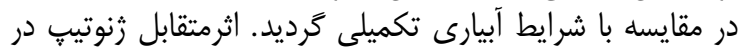

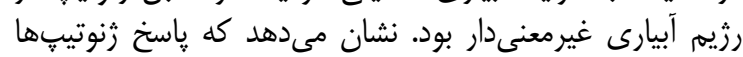

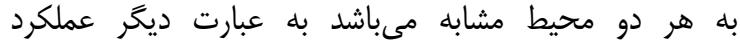

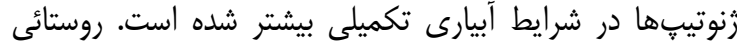

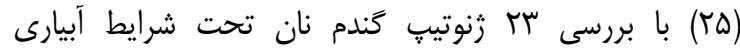

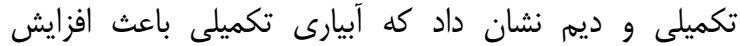

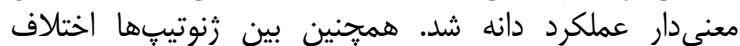

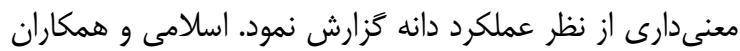

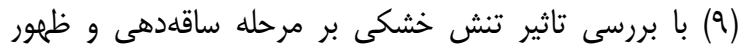

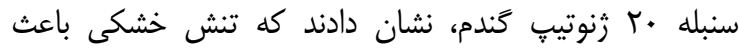

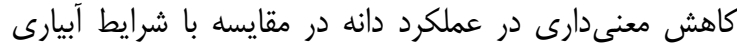

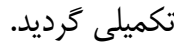

هزار ضدعفونى شدند. بعد از انجام عمليات متداول تهييه زمين

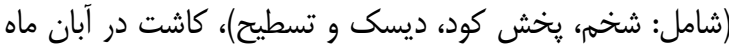

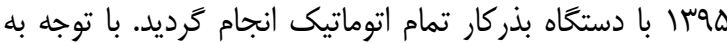

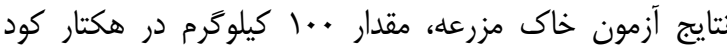

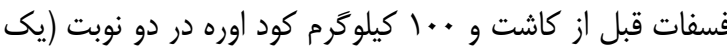

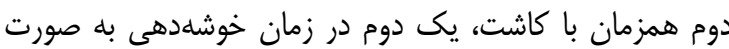

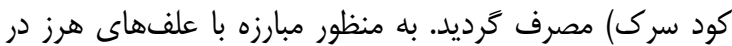

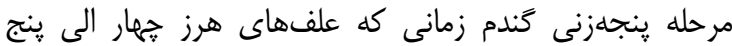

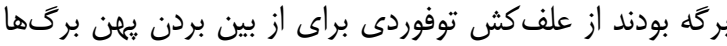

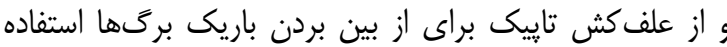

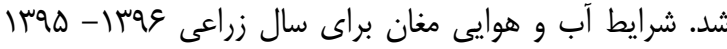

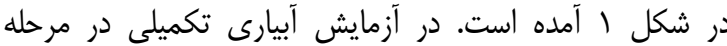

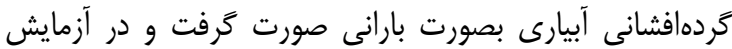

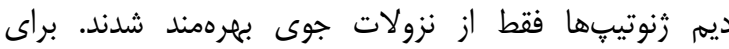

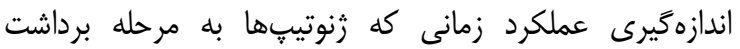

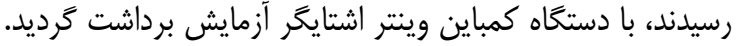

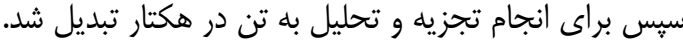

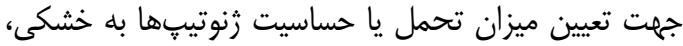

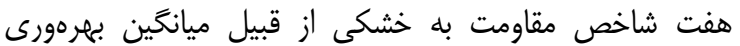

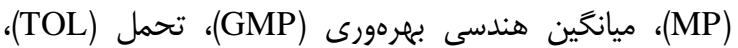

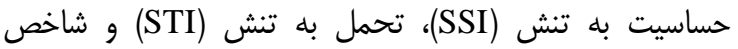

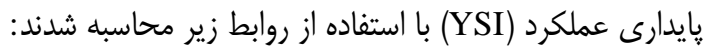

$$
\begin{aligned}
\mathrm{MP} & =\frac{\mathrm{Yp}+\mathrm{Ys}}{2} \\
\mathrm{GMP} & =\sqrt{\mathrm{Yp} \times \mathrm{Ys}} \\
\mathrm{TOL} & =\mathrm{Yp}-\mathrm{Ys} \\
\mathrm{STI} & =\frac{\mathrm{Yp} \times \mathrm{Ys}}{\overline{\mathrm{Y}} \mathrm{p}^{2}} \\
\mathrm{SSI} & =\frac{1-\left(\frac{\mathrm{Ys}}{\mathrm{Yp}}\right)}{1-\left(\frac{\bar{Y} s}{\bar{Y} p}\right)} \\
\mathrm{YSI} & =\frac{\mathrm{Ys}}{\mathrm{Yp}}
\end{aligned}
$$

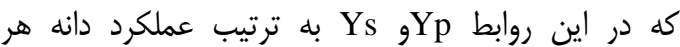

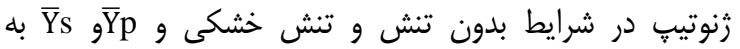

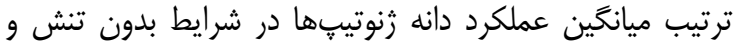

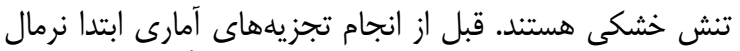

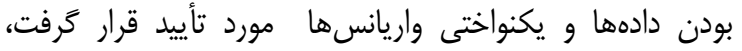

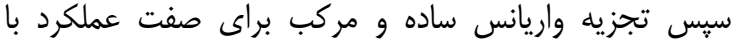

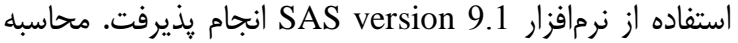


Table 1. Pedigree of bread wheat genotypes used in the experiment

$$
\text { جدول ا- يديخرى (شجره) زنوتيڤهاى گندم نان مورد استفاده در آزمايش }
$$

\begin{tabular}{|c|c|}
\hline Genotype No & Variety / Line \\
\hline 1 & AFTAB \\
\hline 2 & KS82142/2*WBLL1CMSA01Y00397T-040M-040P0Y-040M-030ZTM-040SY-4M-0Y-0SY \\
\hline 3 & KS82142/2*WBLL1CMSA01Y00397T-040M-040P0Y-040M-030ZTM-040SY-21M-0Y-0SY \\
\hline 4 & PASTOR//HXL7573/2*BAU/3/WBLL1PTSS02Y00023S-0Y-030ZTM-040SY-040M-19Y-0M-0SY \\
\hline 5 & $\begin{array}{l}\text { TC870344/GUI//TEMPORALERAM87/AGR/3/2*WBLL1CMSA01Y00725T-040M-040P0Y-040M-030ZTM-040SY-33M-0Y- } \\
\text { OSY }\end{array}$ \\
\hline 6 & KS82W418/SPN/3/CHEN/AE.SQ//2*OPATA/4/FRET2CMSA01M00316T-040Y-040M-030ZTM-040SY-040M-53Y-0M-0SY \\
\hline 7 & WHEAR/KRONSTAD F2004CGSS04Y00106S-099Y-099M- 099Y-099M-13WGY-0B \\
\hline 8 & QIMMA-8 CMSS93Y00332S-1AP-3AP-3AP-0APS-0AP \\
\hline 9 & TC870344/GUI//TEMPORALERAM87/AGR/3/2*WBLL1CMSA01Y00725T-040M-030ZTM-040SY-10M-0Y-0SY \\
\hline 10 & $\begin{array}{l}\text { FRET2*2/4/SNI/TRAP\#1/3/KAUZ*2/TRAP//KAUZ/5/PFAU/WEAVER//BRAMBLINGCMSS05B00480S-099Y-099M-099Y- } \\
\text { 099ZTM-22WGY-0B }\end{array}$ \\
\hline 11 & $\begin{array}{l}\text { KAUZ//ALTAR84/AOS/3/MILAN/KAUZ/4/HUITES } \\
\text { /7/CAL/NH//H567.71/3/SERI/4/CAL/NH//H567.71/5/2*KAUZ/PASTORCMSS05B00581S-099Y-099M-099Y-099ZTM- } \\
\text { 2WGY-0B }\end{array}$ \\
\hline 12 & HAMAM-4/ANGI-2ICW02-00621-2AP/0TS-0AP-0AP-6AP-0AP \\
\hline 13 & ATTILA*2/PBW65//KACHUCMSS05B00004S-099Y-099M-099Y-099ZTM-1WGY-0B \\
\hline 14 & PBW343*2/KUKUNA//SRTU/3/PBW343*2/KHVAKICGSS05B00261T-099TOPY-099M-099NJ-099NJ-6WGY-0B \\
\hline 15 & KACHU\#1/KIRITATI/KACHUCMSS06Y00778T-099TOPM-099Y-099ZTM-099NJ-099NJ-6WGY-0B \\
\hline 16 & $\begin{array}{l}\text { BABAX/KS93U76//BABAX/3/2*SOKOLLCMSA06M00008T-024(PINBD1BHET)Y-040ZTM-026(PINBD1BPOS) ZTY- } \\
\text { 20ZTM-0Y-0B }\end{array}$ \\
\hline 17 & LAKHISH/4/NESTOR/3/HE1/3*CNO79//2*SERIIRBWG-2007-010G-0G-0G-0G-12G \\
\hline 18 & PASTOR/4/NESTOR/3/HE1/3*CNO79//2*SERIIRBWG-2007-022G-0G-0G-0G-1G \\
\hline 19 & GHK"S"BOW"S"//90-ZHONG87/4/NESTOR/3/HE1/3*CNO79//2*SERIIRBWG-2007-049G-0G-0G-0G-14G \\
\hline 20 & GHK"S"BOW"S"//90-ZHONG87/4/NESTOR/3/HE1/3*CNO79//2*SERIIRBWG-2007-049G-0G-0G-0G-19G \\
\hline 21 & BERKUT/MUU//DANPHE \#1CMSA07M00073T-050Y-040ZTM-040ZTY-31ZTM-010Y-02B-0Y \\
\hline 22 & TILILA/JUCHI/4/SERI.1B//KAUZ/HEVO/3/AMADCMSS06Y00868T-099TOPM-099Y-099ZTM-099Y-099M-8WGY-0B \\
\hline 23 & $\begin{array}{l}\text { ROLF07*2/5/REH/HARE//2*BCN/3/CROC_1/AE.SQUARROSA(213)//PGO/4/HUITESCMSS 06Y00926T-099TOPM-099Y- } \\
\text { 099ZTM-099Y-099M-5WGY-0B }\end{array}$ \\
\hline 24 & SUP152/BLOUK \#1CMSS06B00033S-0Y-099ZTM-099NJ-099NJ-3WGY-0B \\
\hline 25 & MUTUS/AKURICMSS06B00451S-0Y-099ZTM-099Y-099M-3WGY-0B \\
\hline 26 & $\begin{array}{c}\text { NAC/TH.AC//3*PVN/3/MIRLO/BUC/4/2*PASTOR/5/KACHU/6/KACHUCMSS06B00734T-099TOPY-099ZTM-099Y-099M- } \\
\text { 13WGY-0B }\end{array}$ \\
\hline 27 & KIRITATI/WBLL1//2*BLOUK \#1CMSS07Y00849T-099TOPM-099Y-099M-099Y-17M-0WGY \\
\hline 28 & BAJ \#1*2/WHEARCMSS07Y01201T-099TOPM-099Y-099M-099Y-15M-0WGY \\
\hline 29 & FRET2*2/BRAMBLING//BECARD/3/WBLL1*2/BRAMBLINGCMSS07B00560T-099TOPY-099M-099Y-099M-19WGY-0B \\
\hline 30 & KACHU/BECARD//WBLL1*2/BRAMBLINGCMSS07B00580T-099TOPY-099M-099Y-099M-10WGY-0B \\
\hline 31 & KA/NAC//TRCH/3/VORB CMSA07M00443S-040M-0NJ-0NJ-10Y-OB \\
\hline 32 & $\begin{array}{c}\text { TACUPETOF2001*2/BRAMBLING/3/ KIRITATI//PBW65/2*SERI.1B/4/ND643/2*WBLL1CMSS08Y00676T-099TOPM-099Y- } \\
\text { 099M-099NJ-099NJ-2WGY-0B }\end{array}$ \\
\hline 33 & $\begin{array}{l}\text { THELIN/3/BABAX/LR42//BABAX/4/BABAX/LR42//BABAX*2/5/KIRITATI/2*TRCHCMSS08Y00756T-099TOPM-099Y- } \\
\text { 099M-099NJ-20WGY-0B }\end{array}$ \\
\hline 34 & MUNAL*2/WESTONIACMSS08Y00833T-099TOPM-099Y-099M-099NJ-099NJ-8WGY-0B \\
\hline 35 & $\begin{array}{l}\text { LERKE/5/KAUZ/3/MYNA/VUL//BUC/FLK/4/MILAN/6/PROGRESOF2007/7/KIRITATI/4/ 2*SERI.1B*2/ } \\
\text { 3/KAUZ*2/BOW//KAUZ CMSS08B00400S-099M-099Y-25M-0WGY }\end{array}$ \\
\hline 36 & KARIM \\
\hline
\end{tabular}

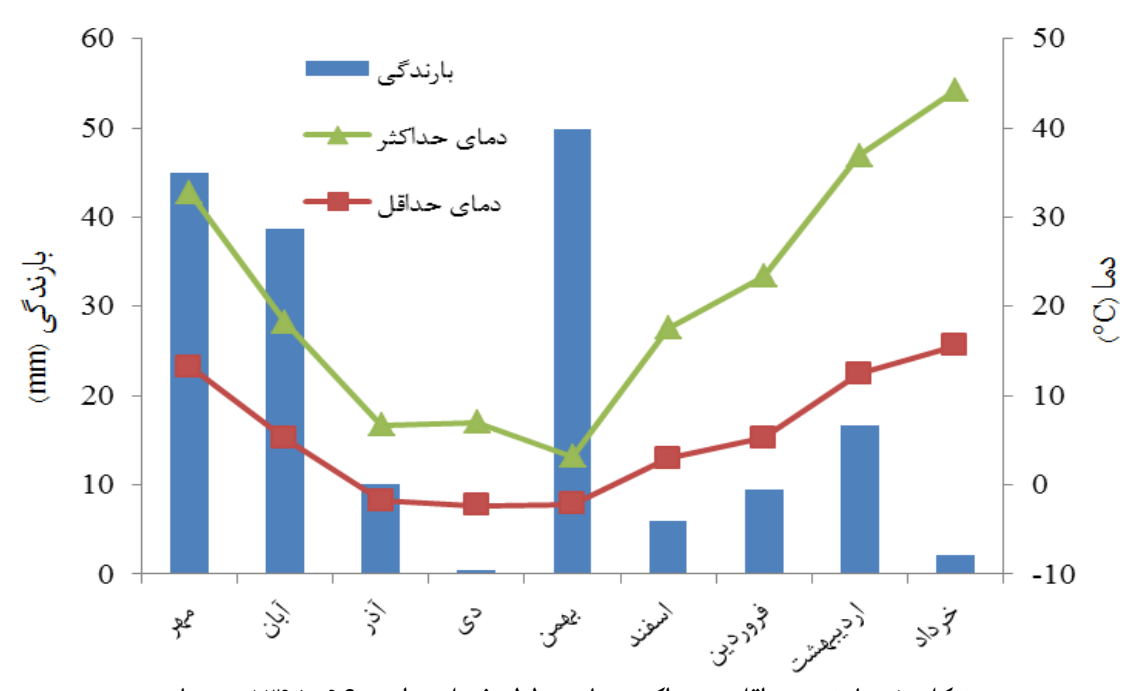

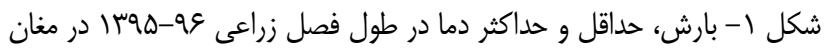

Figure 1. Rainfall and minimum and maximum temperatures recorded during 2016-2017 growing season in Moghan 
جدول r- تجزيه واريانس ساده براى صفت عملكرد دانه در شرايط آبيارى تكميلى و ديم در گندم نان Table 2. Analysis of variance for grain yield of bread wheat in rainfed and supplemental irrigation conditions

\begin{tabular}{|c|c|c|c|}
\hline \multicolumn{2}{|c|}{ ميانخين مربعات } & \multirow[b]{2}{*}{ درجه آزادى } & \multirow[b]{2}{*}{ منابع تغيير } \\
\hline Ys & $\mathrm{Yp}^{\mathrm{a}}$ & & \\
\hline $1191 \mathrm{~V} / \cdot \wedge^{\mathrm{nS}}$ & $V \cdot r / 9 V / V q^{* * *}$ & $r$ & بلوى \\
\hline$r \cdot V \wedge \notin \varepsilon / q)^{*}$ & $r M r \cdot r / r \Lambda^{* * *}$ & ro & رُنوتيب \\
\hline$|T K E| F / \Delta F$ & $|r q 9| \cdot|r|$ & $v$. & اشتباه آزمايشى \\
\hline
\end{tabular}
جدول ب- تجزيه واريانس مركب براى صفت عملكرد دانه در كندم نان

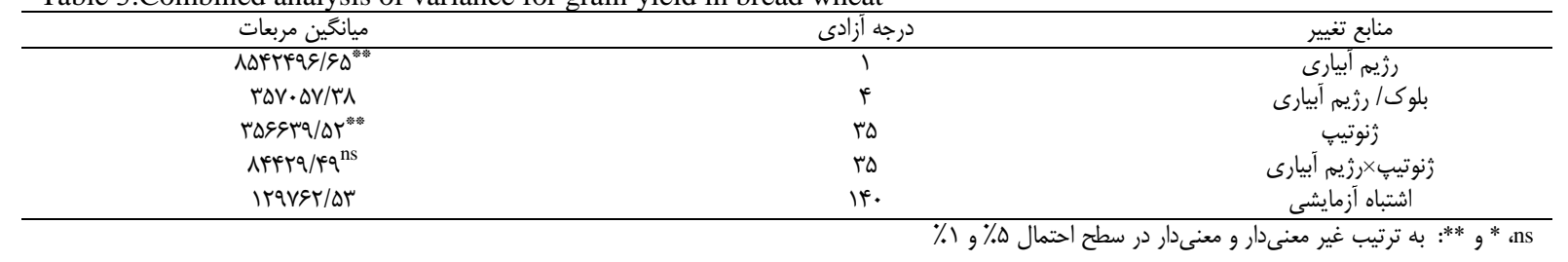

بود. بنابراين رايين بودن شاخص TOL الزاماً به معنى بالا بودن

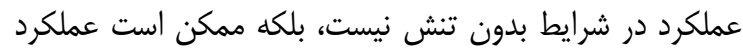

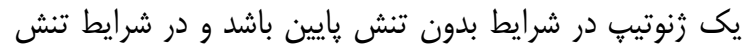

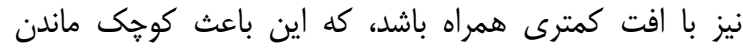
TOL

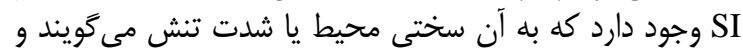

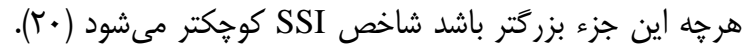

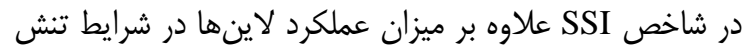

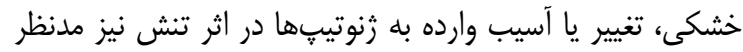

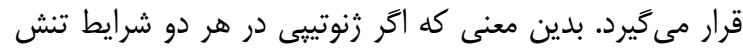

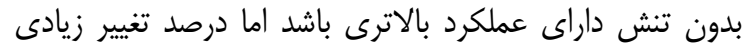

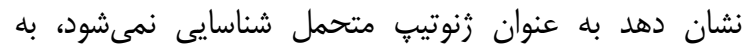

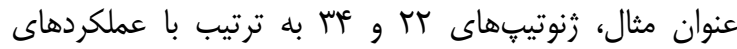

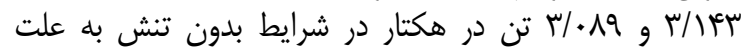

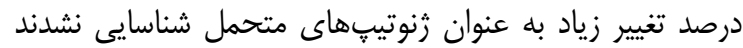

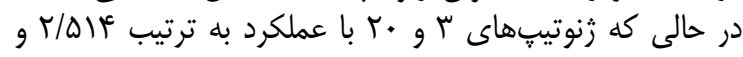

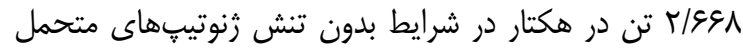

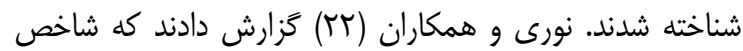

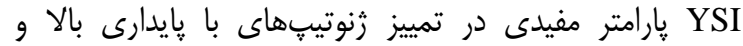

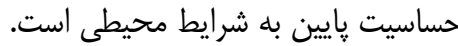

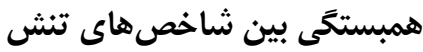

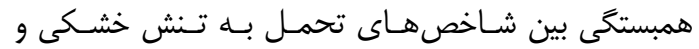

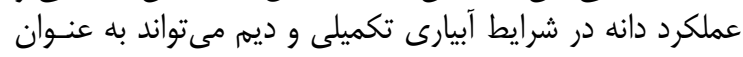

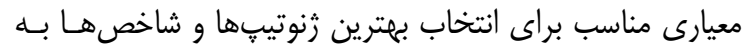

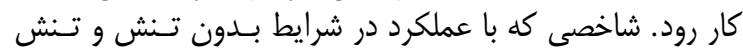

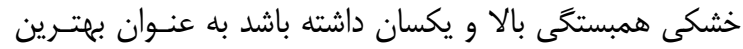

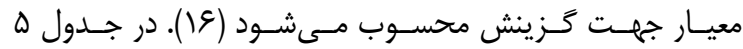

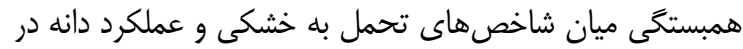

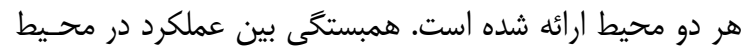

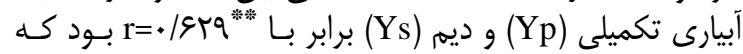

شاخص هاى تحمل تنش خشكى

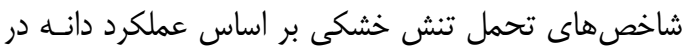

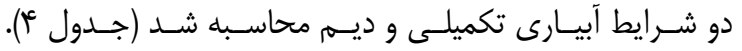

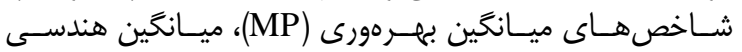

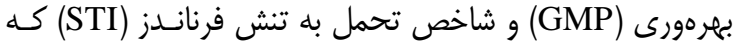

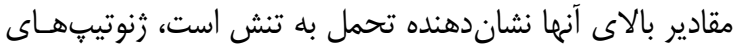

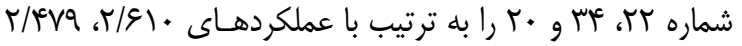

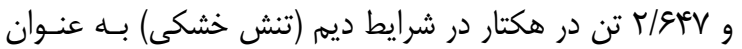

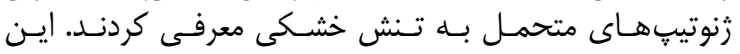

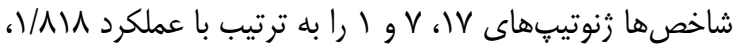

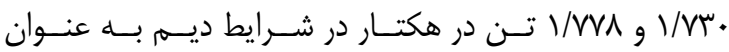

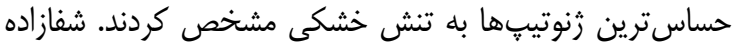

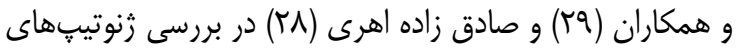

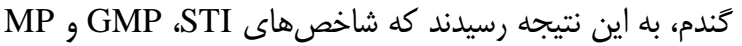

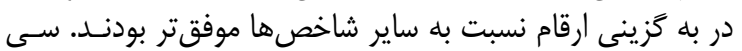

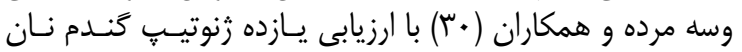

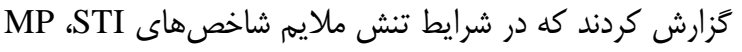

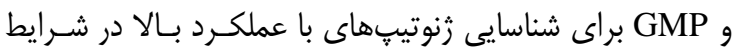
تنش و بدون تنش مناسب هستند. شاخص تحمل (TOL) و شاخص حساسيت به تهن تنش (SSI)

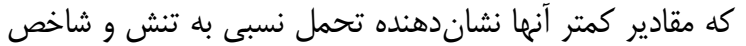

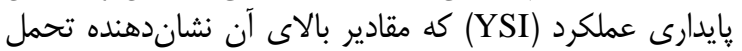

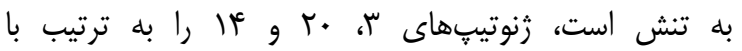

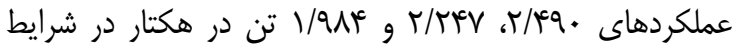

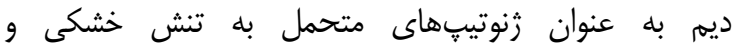

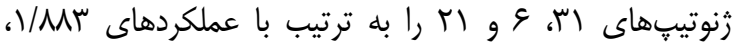

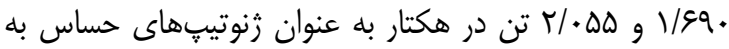

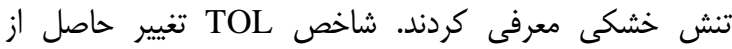

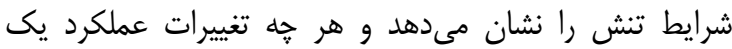

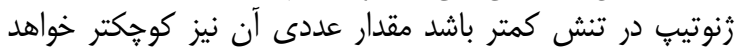


جدول ع- شاخصهاى تحمل تنش خشكى و ميانكين عملكرد دانه رنوتيِهاى كندم در شرايط آبيارى تكميلى و ديم Table 4. Drought tolerance indices and mean of grain yield in wheat genotypes under rainfed and supplemental irrigation conditions

\begin{tabular}{|c|c|c|c|c|c|c|c|c|}
\hline YSI & SSI & TOL & MP & GMP & STI & Ys & $\mathrm{Yp}^{\mathrm{a}}$ & رنوتيبٍ \\
\hline$\cdot / \mathrm{A} \cdot \mathrm{r}$ & 1/TAT &.$/ 44 q$ & $1 / 997$ & $1 / 2 \wedge \Delta$ &.$/ 9 T \Delta$ & $1 / V V A$ & T/TIV & 1 \\
\hline " & ז"Q/. & 年 & $r / \varepsilon q$. & $r / F A T$ & . /qv9 & T/TqR & $r / 8 M$ & $r$ \\
\hline.$/ 991$ & .1 .09 & . 1. KF & $r / \Delta \cdot r$ & $r / \Delta \cdot r$ &.$/ 994$ & $r / \kappa q$. & $r / \Delta / F$ & r \\
\hline . $/ A V^{\mu}$ & $\cdot 11.9$ & זוזן. & $r / T q \Delta$ & $r / r q$. & . & r//rq & $r / F \Delta)$ & f \\
\hline$\cdot / N{ }^{\mu}$ & $1 / v \cdot 9$ &.$/ 9 \vee \Delta$ & $r /|q|$ & T//ra & . MTT & I/ATF & $r / \kappa 99$ & $\Delta$ \\
\hline.$/ 89$. & 1/9.. & $\cdot / V \Delta \Lambda$ & $r / .9 q$ & $r / . r r$ & $\cdot \mid \varnothing \Delta A$ & $1 / 99$. & T/FFA & 8 \\
\hline س &.$/ 491$ & .1 .9$. & $1 / N G T$ & I/AGT & $\cdot / \Delta \Delta$. & $1 / \wedge 1 \wedge$ & $1 / 9 \cdot 1$ & $v$ \\
\hline ./q & . Far & .1199 & T/TVG & r/TVQ & . /NT. & $r / 1 q T$ & T/F & $\wedge$ \\
\hline.$/$ ITA & $1 / V Y Y$ &.$/ 8 \wedge \Delta$ & $r / \mid V I$ & T/MFE & . VYA & 1/Arq & T/D/T & $q$ \\
\hline - Mate &.$/ Q \wedge 8$ & . & $r / r \cdot 1$ & $r / 19 \mu$ & . IVGY & $r / \cdot 10$ & T/rAV & 1. \\
\hline - MAT & - /VEa &.$/ T r K$ & T/IVT & $r / / \& \Lambda$ & / / NeQ & צr/F & $r / r \cdot \Lambda$ & 11 \\
\hline - /NFa &.$/ 911$ & & $r / .99$ & $r / .91$ & r/qם/. & $1 / 194$ & T/TVD & $\pi$ \\
\hline -/vas & $1 / \% \wedge 9$ & . /49q & r/IVG & T/MGT & $\left.\cdot / N{ }^{4}\right)$ & $1 / 949$ & אזא/T & r \\
\hline.$/ 941$ & $\cdot|\backslash \Lambda|$ & $\cdot / \cdot \Delta \Lambda$ & $r / \cdot \pi$ & $r / .1 \mu$ & . MET & 1/40F & T/.FT & $1 f^{f}$ \\
\hline . &.$/ 9 Y 1$ &.$/ 410$ & T/QTK & $r / 8 / 0$ & $1 / \cdot 1 e^{c}$ & $T / F \mid G$ & r/ArI & 10 \\
\hline$\cdot|\wedge| s$ & I/IGT &.$/ 499$ & $r / \Psi, V$ & $r / \Gamma \cdot \omega$ & . MET & $r / \wedge r$ & $r(\Delta Q)$ & 19 \\
\hline . Mat &.$/ N \varepsilon$ &.$/ T V Y$ & $1 / N \& 9$ & $1 / A 81$ &.$/ \Delta F q$ & $1 / N{ }^{\mu}$. & $r / . . r$ & IV \\
\hline.$/ 190$ & .1990 & gr/rq & $r / .90$ & $r / . q T$ &.$/ 8 V{ }^{6}$ & 1/9ఎ. & T/M. & $M$ \\
\hline ./VAD & سצאץ/1 &.$/ 810$ & $r / \Delta F \Lambda$ & $r / \Delta r$. & $1 / .14$ & T/MFI & T/ADS & 19 \\
\hline.$/ 910$ & .1 .90 & $\cdot / \cdot F$ & $r / 99 V$ & $r / 99 V$ & I/TTV & $r / \mathscr{Q})^{4} V$ & $r / 8 M$ & $r$. \\
\hline$\cdot / V \cdot r$ & I/AVA & $\cdot / N \& V$ & $T / \mathcal{A M}$ & $r / F \Delta$. &.$/ 901$ & $\tau / \cdot \Delta \Delta$ & T/QTI & r \\
\hline . $/ \Lambda \mu$. & $.1 \cdot v^{c}$ & r & T/AVG & T/MAY & $1 / \mu_{.}$ & $r / 8)$. & שאו/T & rT \\
\hline - /qDF & - /rat &.$/ 11$. & $r / \mu \cdot \Delta$ & $r / \Gamma \cdot \Delta$ & - MFT & $r / T \Delta$. & T/ץq. & זr \\
\hline.$/ 909$ & . Tq. & .1 .99 & $r / T \Delta T$ & $r / T \Delta T$ & $\cdot /$ AVG & $r / \mu \cdot r$ & $r / F+1$ & $r F$ \\
\hline - /NFA &.$/ 911$ & $\cdot / 4 \cdot r$ & r/rq & 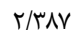 &.$/ 9 \cdot r$ & r/lqq & r/QQV & $r \Delta$ \\
\hline$\cdot / 1 \cdot 1$ & I/TIT &.$/ 019$ & 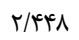 & T/FMY & q & T/119 & $T / V \cdot V$ & Tq \\
\hline.$/ 941$ & . IAT &.$/ \cdot v g$ & $r / \Delta Q Q$ & $r / \Delta Q Q$ & $1 / .91$ & $r / \Delta \Delta \Lambda$ & T/GHY & Tr \\
\hline.$/ 67 q$ & $1 / 491$ & . & T/R\&D & T/MFV & $\cdot / \Lambda_{*} \cdot$ & 1/a人r & T/DFS & $r \Lambda$ \\
\hline$\cdot|\Lambda|$ & $1 / T .$. & . kapt & T/Tr. & $r / \lambda \cdot \Lambda$ & $\cdot / v \cdot F^{c}$ & $1 / 194$ & T/MFT & rq \\
\hline.$/ W r$ & $1 / F \mu r y$ & $\cdot / \Delta \wedge V$ & r/TqA & $r / r v q$ & rAT/. & $r / . . F$ & $r / \Delta q)$ & r. \\
\hline$\cdot|8 \wedge|$ & $r / . r)$ & /MMr & T/MTQ & T/TAT & . /ATa & I/MAr & T/VGG & r \\
\hline$\cdot / V F A$ & 1/ब9V & 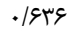 & $T / T \cdot T$ & $r / I V q$ & - IVQT & $1 / M F$ & $r / \Delta / 9$ & r \\
\hline - / $\triangle \Delta S$ &.$/ 9.9$ & $\cdot / 4 \cdot 1$ & r/ब & r/qu & $1 / .98$ & T/FTr & T/AFT & זس \\
\hline$\cdot / \mathrm{A} \cdot \mathrm{r}$ & $1 / T \Delta$. &.$|9|$ & T/VAr & T/VGV & $1 / 4 I F$ & r/Frq & $r / \wedge q$ & re \\
\hline$\cdot / \Lambda \Delta \Delta$ &.$/ 919$ & צ צr/. & $T / / Q T$ & T/NFD & ./VYq & $1 / 945$ & $T / \pi / q$ & ro \\
\hline.$/ 9 \Delta \Delta$ & . trat & . & $T / F A F$ & $r / F \Delta F$ &.$/ 9 \Delta F^{\circ}$ & r/raی & $T / \Delta)$. & g \\
\hline
\end{tabular}

Table 5. Correlation coefficient between drought tolerance indices and grain yield under rainfed and supplemental

\begin{tabular}{|c|c|c|c|c|c|c|c|c|}
\hline YSI & SSI & TOL & MP & GMP & STI & Ys & $\mathrm{Yp}^{\mathrm{a}}$ & \\
\hline & & & & & & & $1 / \cdots$ & $Y p$ \\
\hline & & & & & & $1 / \ldots$ & $.1949^{* \omega^{*}}$ & Ys \\
\hline & & & & & $1 / \ldots$ &.$/ 910^{* * *}$ & $\cdot \mid M \mathcal{F}^{* *: 3}$ & STI \\
\hline & & & & $1 / \ldots$ &.$/ 991^{* * *}$ &.$/ 91 r^{*}$ &.$(19)^{* *}$ & GMP \\
\hline & & & $1 / \ldots$ & ./99.** &.$/ 99 V^{* * 3}$ &.$/ 190^{* * *}$ & $. / 91 . *$ & MP \\
\hline & & $1 / \ldots$ & $\cdot / \cdot 98^{115}$ & . $/ \cdot \Delta r^{113}$ & $\cdot 1 \cdot 4 \Delta^{115}$ & $-\cdot / \Gamma \Delta Q^{*}$ & $\cdot 10 . .{ }^{* n}$ & TOL \\
\hline & $1 / \ldots$ &.$/ 94 r^{* * *}$ & - & $-\cdot 11 \cdot 9^{113}$ &.$- / 110^{113}$ & $-\cdot / \Delta \cdot 1^{* * * a}$ & $\cdot / \Gamma \Delta r^{*}$ & SSI \\
\hline $1 / \cdots$ & $1 / \ldots^{* * *}$ & $-\cdot / 9 \wedge \mu^{* * *}$ & $1.94^{115}$ &.$/ 11.115$ &.$/ 110^{115}$ & 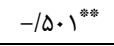 & $-\cdot / \Gamma \Delta \varphi^{* *}$ & YSI \\
\hline
\end{tabular}

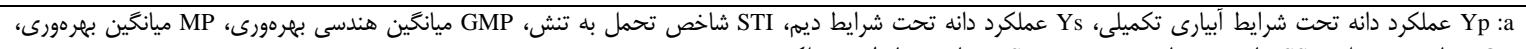

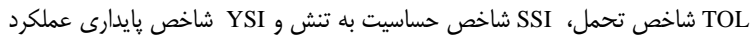

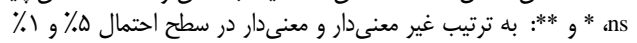




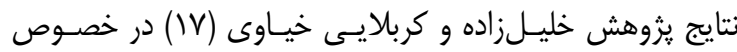

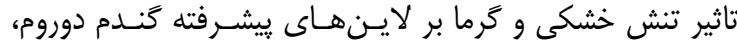

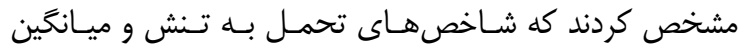

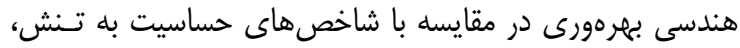

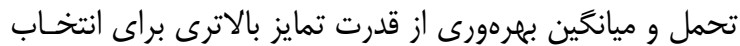

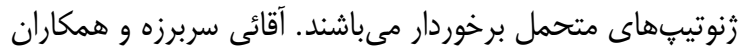

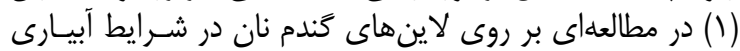

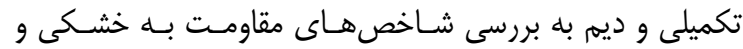

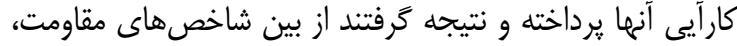

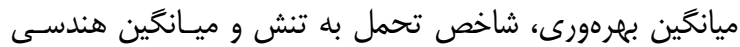

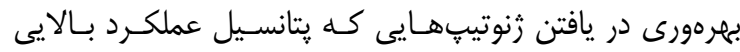

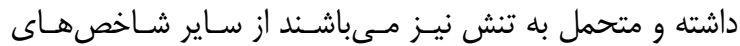

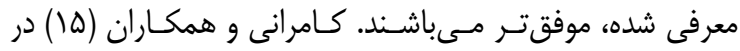

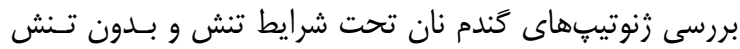

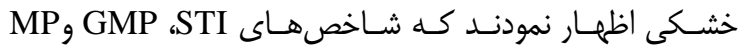

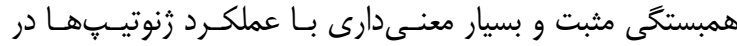

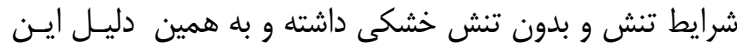

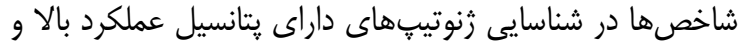

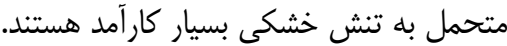

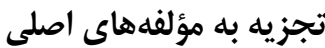

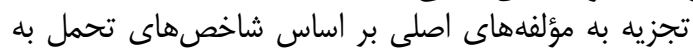

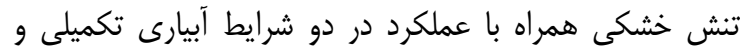

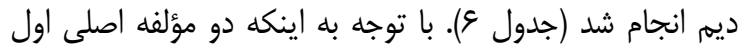

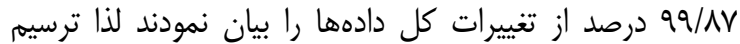

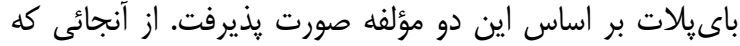

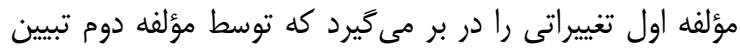

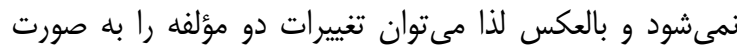

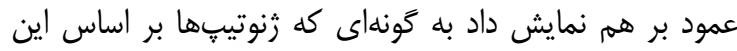

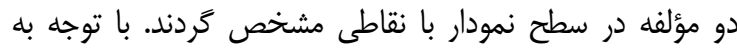

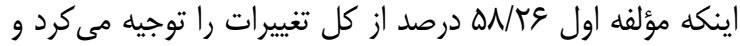

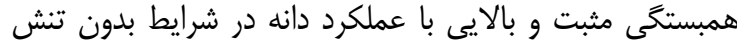
(YP)

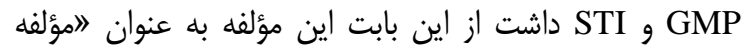

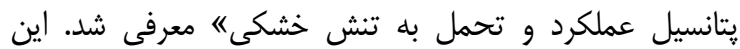

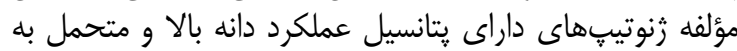

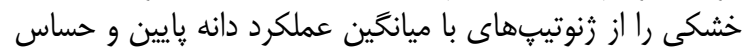

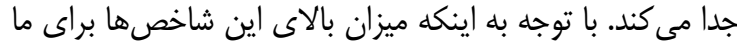

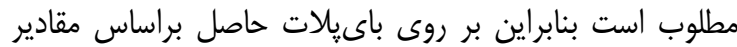

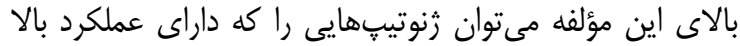

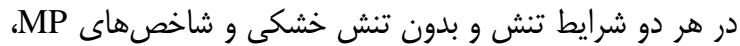

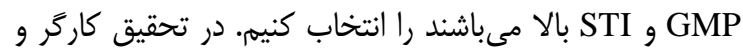

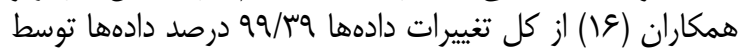

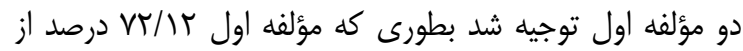

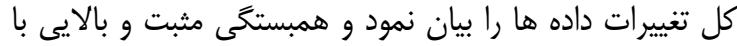

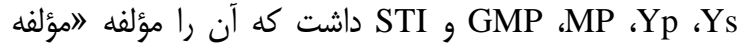
يتانسيل عملكرد و تحمل به تنش خشكى" معرفى كردند.

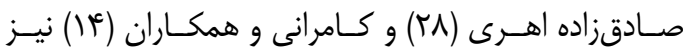

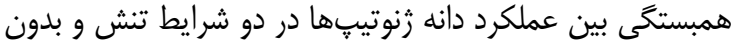

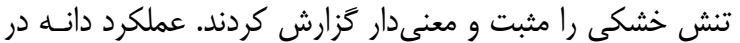

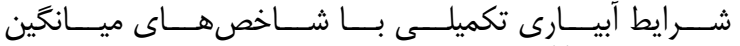

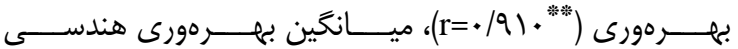

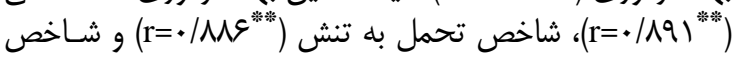
تحمـل (

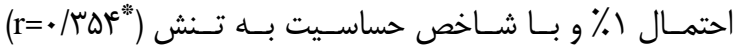

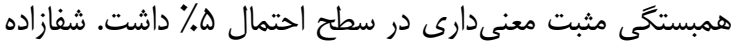

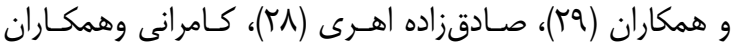

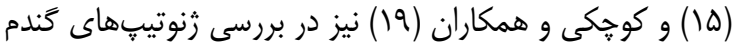

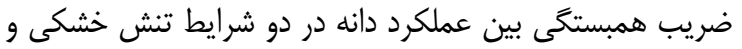

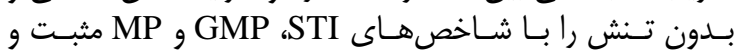

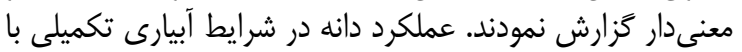

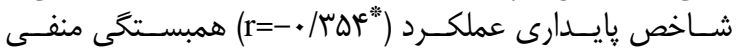

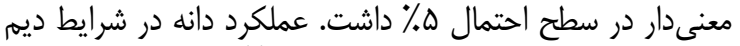

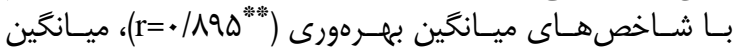

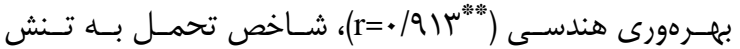

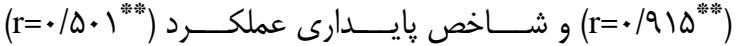

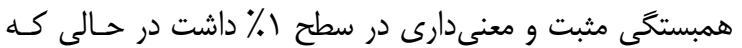

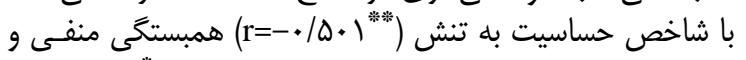

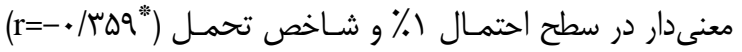

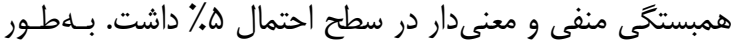

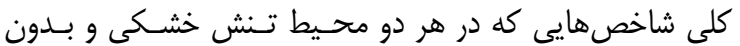

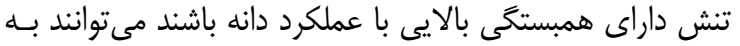

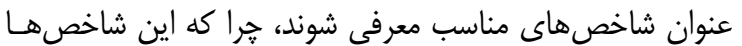

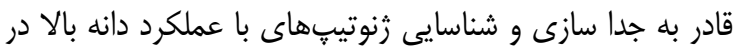

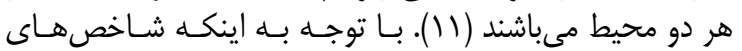

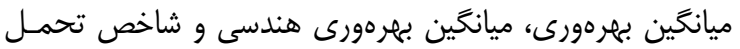

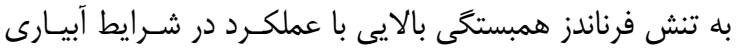

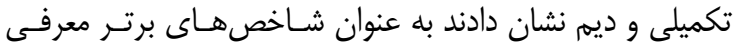

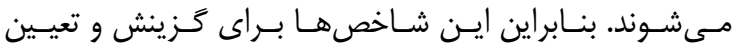

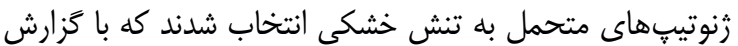

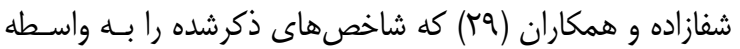

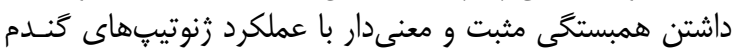

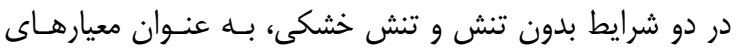

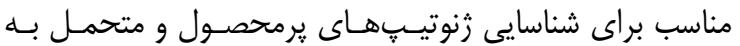

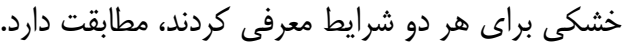

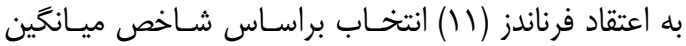

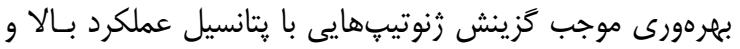

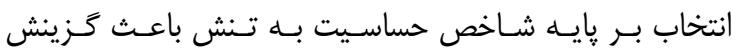

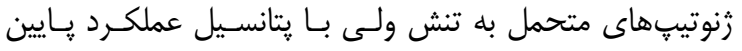

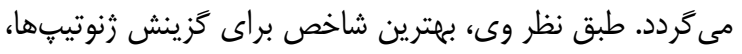

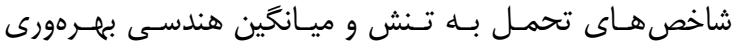

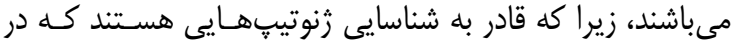
هر دو محيط تنش و بدون تنش عملكرد بالايى توليد مسى كنتــد. 
جدول و- نتايج حاصل از تجزيه به مؤلفهاى اصلى براى شاخصهاى تحمل به تنش و عملكرد دانه در شرايط آبيارى تكميلى و ديم Table 6. Principal components analysis based on drought tolerance indices and grain yield under rainfed and supplemental irrigation conditions

\begin{tabular}{|c|c|c|c|c|c|c|c|c|c|c|c|}
\hline YSI & SSI & TOL & MP & GMP & STI & Ys & $\mathrm{Yp}^{\mathrm{a}}$ & درواريانس تجمعى & درصد واريانس & مقادير ويثه & مؤلفه \\
\hline .11 .9 & $-\cdot / 1 \cdot 9$ & - & . & . & . TES & Drd & ./rqv & $\Delta N / T \& \Lambda$ & $\Delta N / r \& \Lambda$ & $4 / 991$ & 1 \\
\hline r. & IDT & . $/ \Delta F \Delta$ & $.1 \cdot 14$ & .1 .91 & $\% \cdot \Delta V$ &.$- / 19 V$ & $\cdot / r \cdot 9$ & 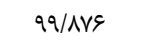 & $|c| / \varepsilon \cdot 1$ & 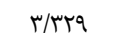 & r \\
\hline$-. / 4+9$ & $-. / 4+9$ & $\cdot \mid 9 \Delta$ & $-\cdot / \cdot r$. & $-\cdot / 1 \cdot r$ & $\cdot / \cdot v V$ & $-\cdot / \mu r$. & - MFE & ११/१८१ &.$/ .14$ & $\cdot / \cdot v$ & $r$ \\
\hline-.1 .9 & .1 .9 & $-\cdot / \cdot v 1$ &.$- /$ Kat &.$- / 1 \mathrm{Vr}$ & $\cdot / M$ & $-\cdot / r \mid a$ & $-\cdot /$ TQQ & १९/११४ & .1 .4$. & . & r \\
\hline.$-|\cdot r|$ &.$/ \cdot 4$ & سא.|.- &.$/ 4 \cdot 9$ & - & .1 .94 &.$/ \% \cdot 9$ & . MFA & $1 .$. &.$/ . .1$ &.$/ . .1$ & $\Delta$ \\
\hline
\end{tabular}


و اين شاخصها بيشتر از •9 (Ys)

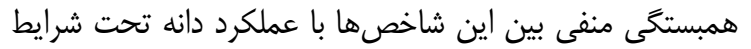

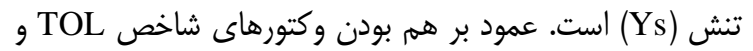

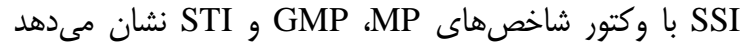

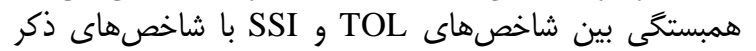

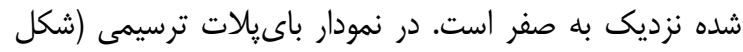

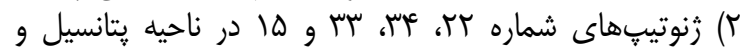

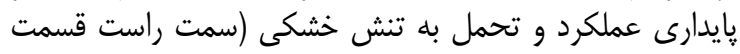

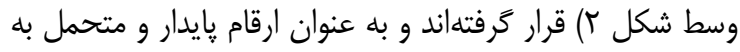

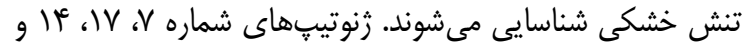

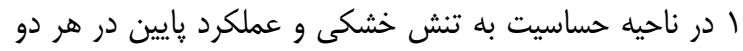

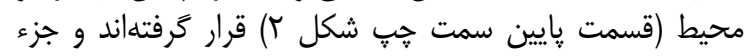

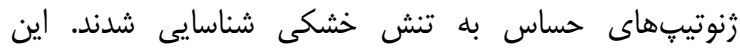

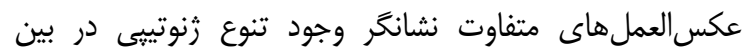

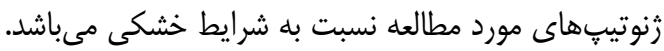

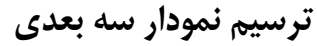

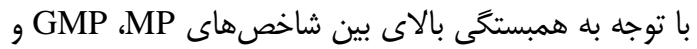

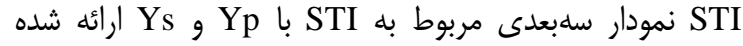

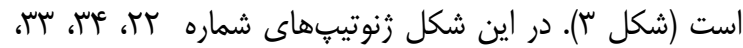

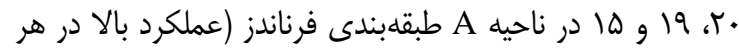

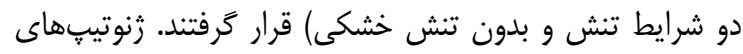

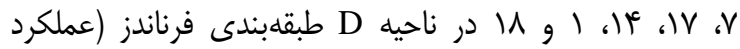

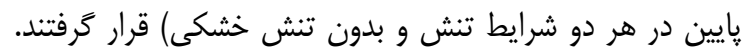

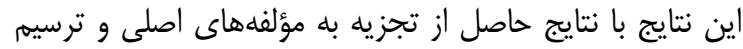

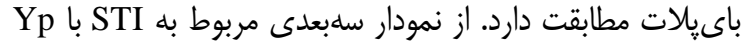
بو براى تشخيص زنوتيبهاى خروه A

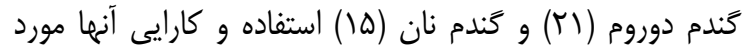
تاييد شده است.

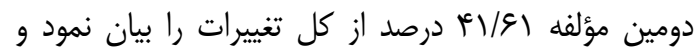

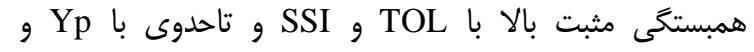

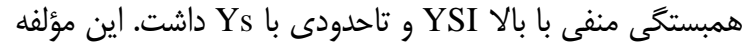

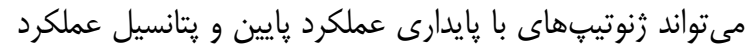

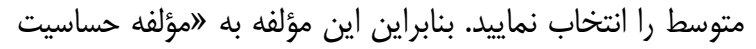

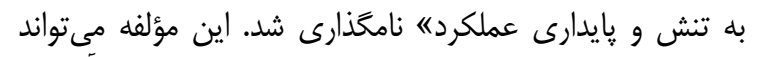

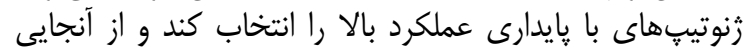

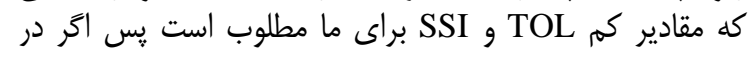

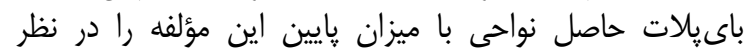

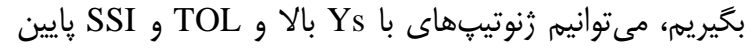
را انتخاب كنيه.

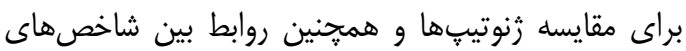

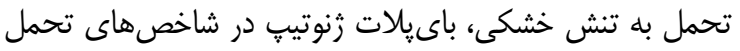

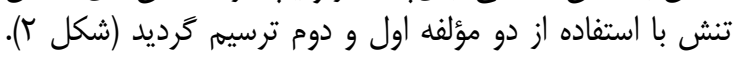

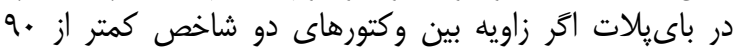

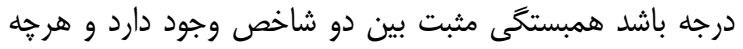

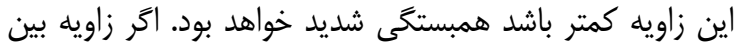

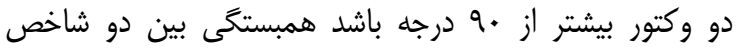

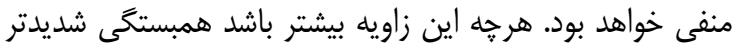

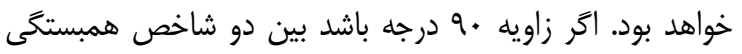

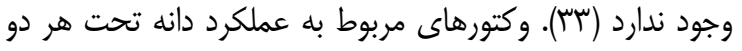

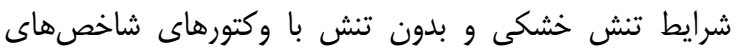

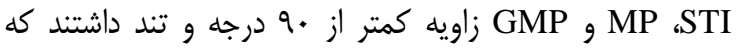

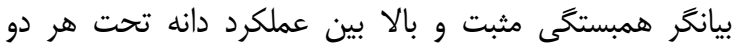

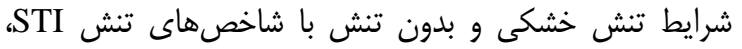

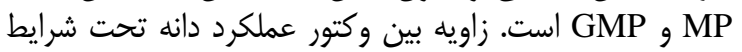

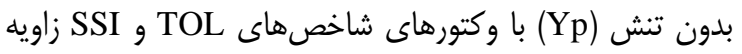

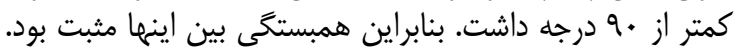
در حالى كه زاويه بين وكتور عملكرد دانه تحت شرايط تن تنش اينش 


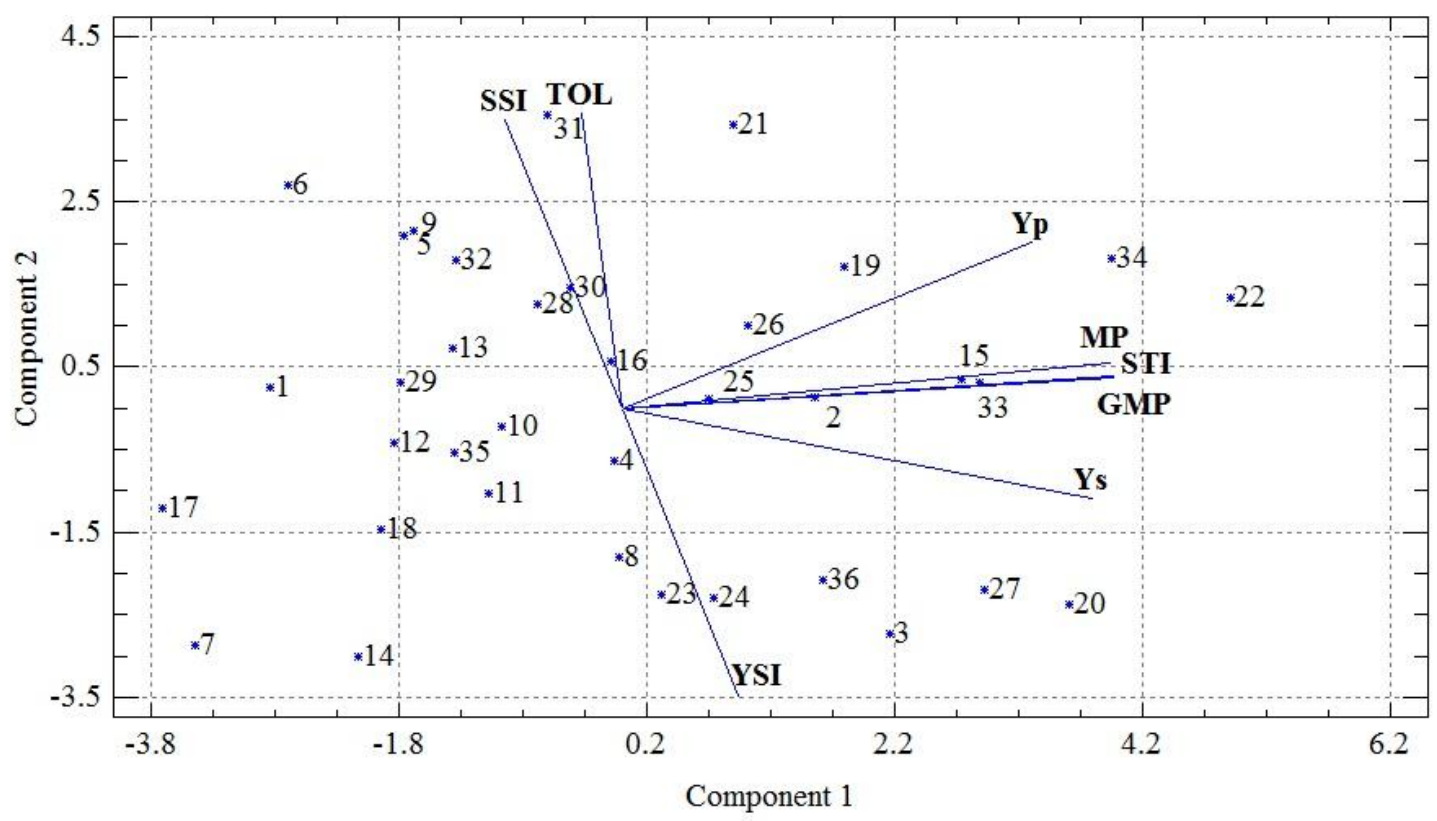

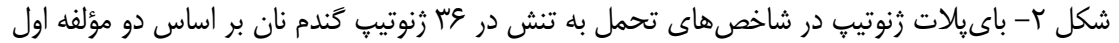

Figure 2. Biplot for drought resistance indices in 36 genotypes of bread wheat based on first two components

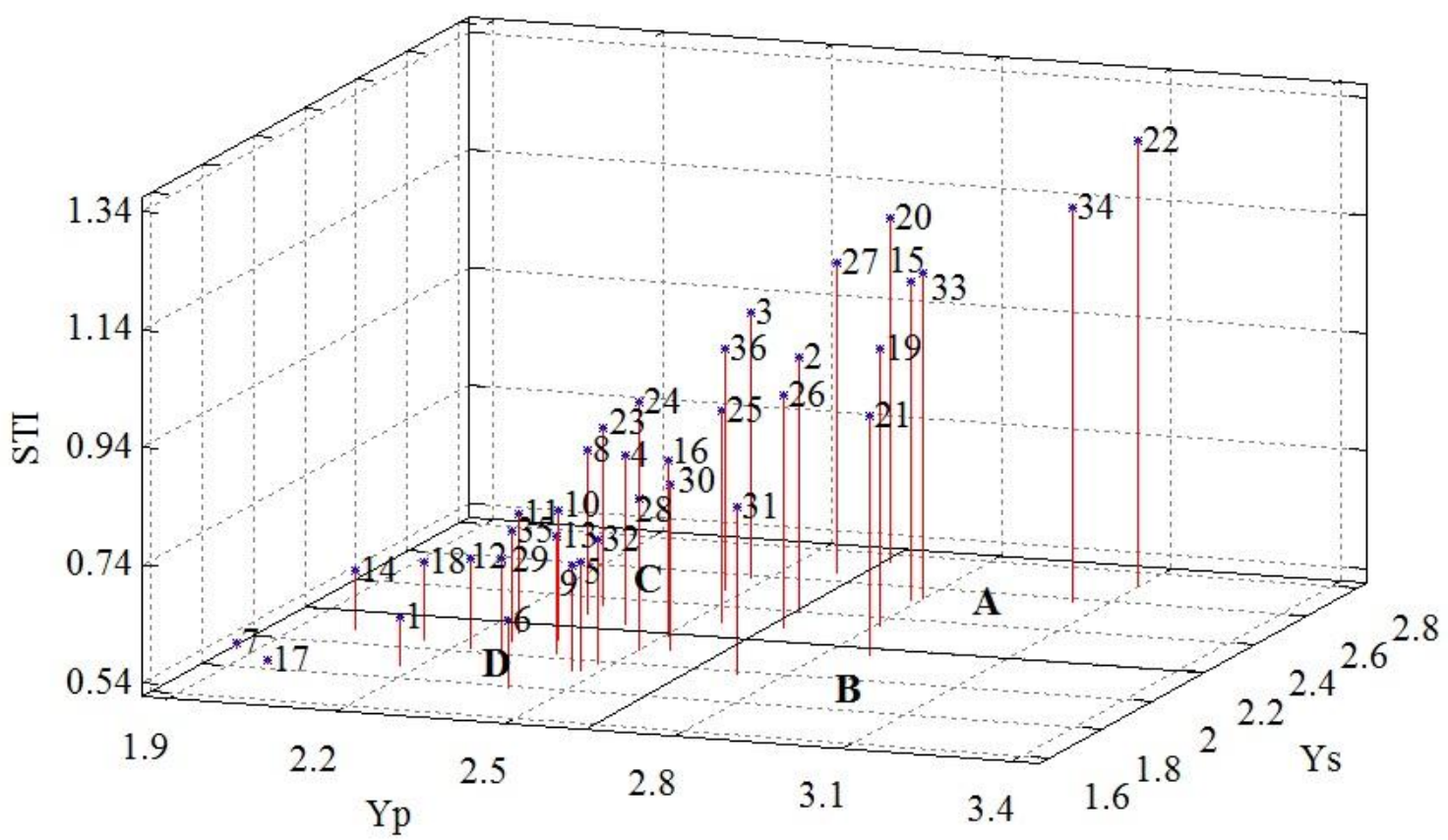

شكل س- نمودار سه بعدى براى شناسايى زنوتيیهاى متحمل به تنش خشكى با استفاده از عملكرد دانه در شرايط تنش (Ys)، بدن تنش (Yp) و شاخص تحمل به تنش (STI)

Figure 3. Three dimensional graph for selection of drought tolerant genotypes using grain yield under stress (Ys) and nonstress (Yp) conditions and stress tolerance index (STI) 
عنوان گروه حساس بله تنش خشكى شناخته مى دُشوند.

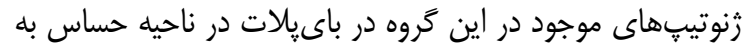

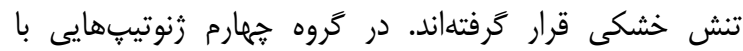

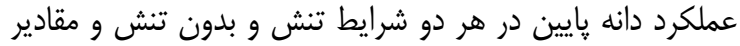

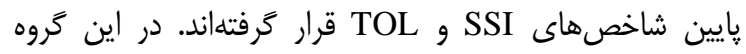

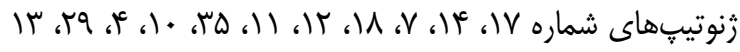

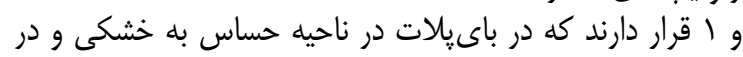

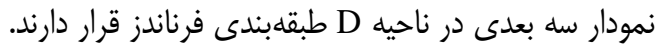

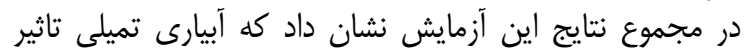

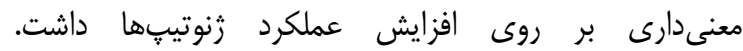

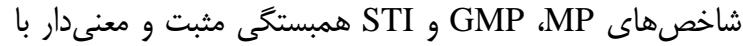

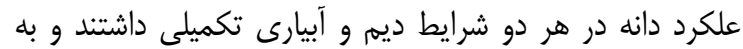

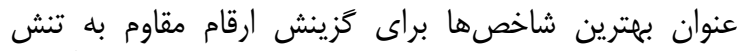

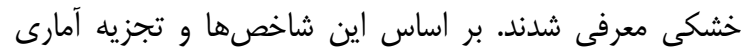

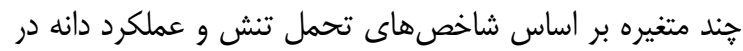

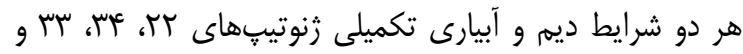

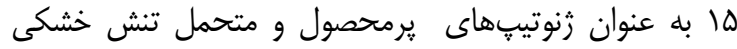

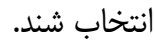

تجزيه خوشهاى

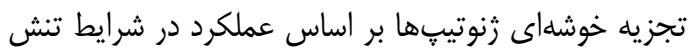

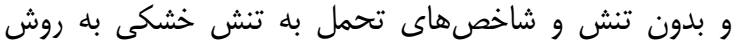
Ward's

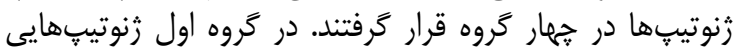

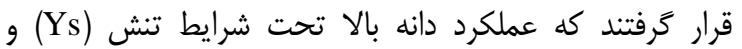

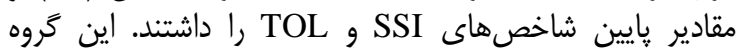

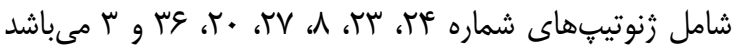

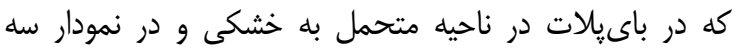

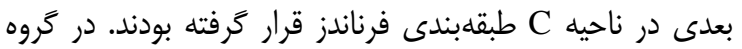

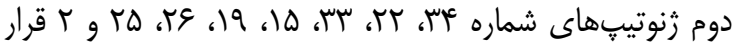

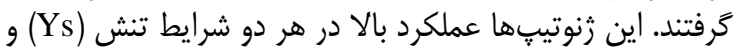

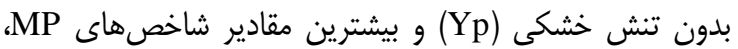
و و GMP

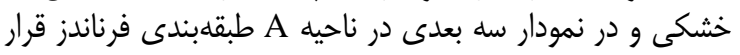

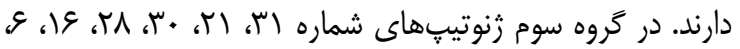

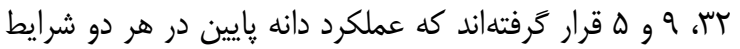
تنش و بدون تنش و مقادير بالاى SSI و TOL قرار دار دارند و و باريه

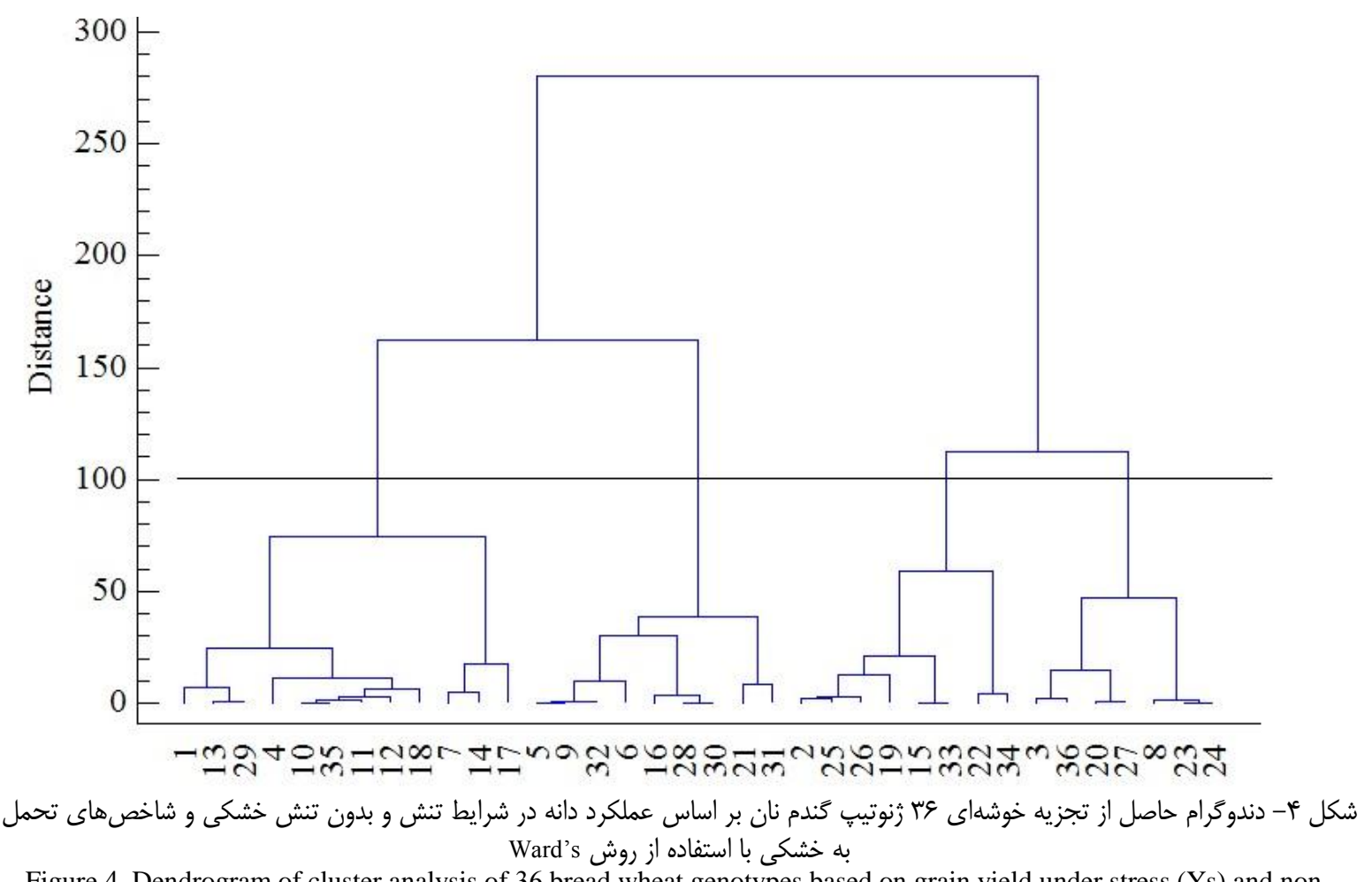

Figure 4. Dendrogram of cluster analysis of 36 bread wheat genotypes based on grain yield under stress (Ys) and nonstress (Yp) conditions and drought tolerance indices using Ward's method 
1. Aghaee Sarbarzeh, M., M. Rostaee, R. Mohammadi, R. Haghparast and R. Rajabi. 2009. Determination of drought tolerant genotypes in bread wheat. Electronical journal of crop production, 2(1): 1-23 (In Persian).

2. Aghaee-Sarbarzeh, M. and M. Rostaee. 2008. Responses genotypes advanced bread wheat to drought stress conditions in cold and cold temperate. Abstracts of 10th Iranian Congress of Crop Production and Plant Breeding, 18-20, August. Karaj- Iran, 217 pp (In Persian).

3. Blum, A. 1988. Plant breeding for stress environments. CRC, Inc., pp: 43-77.

4. Blum, A. 2005. Mitigation of drought stress by crop management. Available online at: http://www.plant stress.com.

5. Bouslama, M. and W.T. Schapaugh. 1984. Stress tolerance in soybean. I: Evaluation of three screening techniques for heat and drought tolerance. Crop Science Journal, 24: 33-937.

6. Calhoum, D.S., C. Gebeyehu, A. Miranda, S. Rajaram and M. Van Ginkel. 1994. Choosing evaluation environments to increase grain yield under drought conditions. Crop Science, 34: 673-678.

7. Chaves, M.M. and M.M. Oliveira 2004. Mechanisms underlying plant resilience to water deficits: Prospects for water-saving agriculture. Journal of Experimental Botany, 407: 2365-2385.

8. Dhanda, S.S. and G.S. Sethi. 2002. Tolerance to drought stress among selected Indian wheat cultivars. Journal of Agricultural Science, 139: 319-326.

9. Eslami R., M. Tajbakhsh, A.A. Ghafari, M. Roustaei and I. Barnousi. 2011. Evaluation of drought tolerance in drylands wheat genotypes under different moisture conditions. Electronical journal of crop production, 5(2): 129-143 (In Persian).

10. Falconer, D.S. 1990. Selection in different environments: effects on environmental sensitivity and on mean performance. Genetics Research, 56: 57-70.

11. Fernandez, G.C. 1992. Effective selection criteria for assessing plant stress tolerance. Proceeding of the international symposium on adaptation of vegetable and other food crops to temperature water stress, 1318, August. Taiwan. pp: 257.

12. Fischer, R.A. and R. Maurer. 1978. Drought resistance in spring wheat cultivars. I. Grain yield responses. Australian Journal of Agricultural Research, 29: 897-912.

13. Ilker, E., O. Tartar, F. Aykut-Tonk and M. Tosun. 2011. Determination of tolerance level of some wheat genotypes to post-anthesis drought. Turkish Journal of Field Crop, 16(1): 59-63.

14. Kamrani, M., A. Farzi and A. Ebadi. 2015. Evaluation of grain yield performance and tolerance to drought stress in wheat genotypes using drought tolerance indices. Cereal Research, 5(3): 231-246 (In Persian).

15. Kamrani, M., A. Farzi and M. Shiri. 2017. Evaluation of Drought Tolerance in Some wheat Genotypes using Drought Tolerance Indices. Journal of Crop Breeding, 9: 9-17 (In Persian).

16. Kargar, S.M.A., M.R. Ghannadha, R. Bozorgi-Pour and H.A. Babaei. 2004. An investigation of drought tolerance indices in some soybean genotypes under restricted irrigation conditions. Iranian Journal of Agricultural Science, 35(1): 129-142 (In Persian).

17. Khalilzade, G.H. and H. Karbalai-Khiavi. 2002. Investigation of drought and heat stress on advanced lines of durum wheat. $7^{\text {th }}$ Iranian congress of crop sciences. 24-26, August. karaj, Iran, pp: 563.

18. Kobota, T.J., A. Palta and N.C. Turner. 1992. Rate of development of postanthesis Water deficits and grain filling of Spring Wheat. Crop Science, 32: 1238-1242.

19. Koocheki, A.R., A. Yazdansepas and H.R. Nikkhah. 2005. Effects of terminal drought on grain yield and some morphological traits in wheat (Triticum aestivum L.) genotypes. Iranian Journal of Crop Sciences, 8(1): 14-29.

20. Moghaddam, A. and M. Hadizadeh. 2002. Response of corn (zea mays L.) hybrids and their parental lines to drought using different stress tolerance indices. Seed and Plant, 18(3): 255-272 (In Persian).

21. Mohammadnia, S., A. Asghari, O. Sofalian, H. Mohammaddoust-ChamanAbad and R. Karimizadeh. 2016. Evaluation of Durum Wheat Lines using Drought Stress Indices. Journal of Crop Breeding, 14: 4153 (In Persian).

22. Nouri, A., A. Etminan, J.A. Teixeira da Silva and R. Mohammadi. 2011. Assessment of yield, yieldrelated traits and drought tolerance of durum wheat genotypes (Triticum turjidum var. durum Desf.), Australian Journal of Crop Science, 5(1): 8-16.

23. Oweis, T. 1997. Supplemental Irrigation: A highly efficient water-use practice. International Center for Agricultural Research in the Dry Areas (ICARDA), Aleppo, Syria.

24. Passioura, J. 2007. The drought environment: physical, biological and agricultural perspectives. Journal of Experimental Botany, 2: 113-117.

25. Roostaei, M. 2015. Effect of Supplementary Irrigation on Grain Yield and some Agronomic Traits of Bread Wheat Genotypes in Maragheh Conditions of Iran. Seed and Plant Improvement Journal, 1-31: 205225 (In Persian).

26. Rosielli, A. and J. Hamblin. 1981. Theoritical aspects of selection for yield in stress and non-stress environment. Crop Science, 21: 943-946.

27. Saba, J., M. Moghaddam, K. Ghassemi and M.R. Nishabouri. 2001. Genetic properties of drought resistance indices. Journal of Agricultural Science and Technology, 3: 43-49 (In Persian).

28. Sadeghzade-Ahari, D. 2006. Evaluation of drought stress tolerance in durum elite wheat genotypes. Iranian Journal of Agricultural Science, 8: 30-45 (In Persian). 
29. Shafazadeh, M., A. Yazdan Sepas, A. Amini and M. Ghanadha. 2004. Study of terminal drought tolerance in promising winter and facultative wheat genotypes using stress susceptibility and tolerance indices. Seed and Plant, 20(1): 57-71 (In Persian).

30. Sio-Se Mardeh, A., A. Ahmadi, K. Poustini and V. Mohamadi. 2006. Evaluation of drought resistance indices under various environmental conditions. Field crop Research, 98: 222-229.

31. Stone, L.R. and A.J. Schlegel. 2006. Yield-water supply relationships of grain sorghum and winter wheat. Agronomy journal, 98: 1359-1366.

32. Vij, S. and A.K. Tyagi. 2007. Emerging trends in the functional genomics of the abiotic stress response in crop plants. Journal of Plant Biotechnology 5: 361-380.

33. Yan, W. and M.S. Kang. 2003. GGE Biplot Analysis: A Graphical Tool for Breeders, Geneticists and Agronomists. CRC Press LLC, Boca Roton, Florida. 


\title{
Identification of Drought Tolerant Genotypes in Dryland Wheat using Drought Tolerance Indices
}

\author{
Morteza Kamrani $^{1}$, Asghar Mehraban ${ }^{2}$ and Manouchehr Shiri ${ }^{3}$

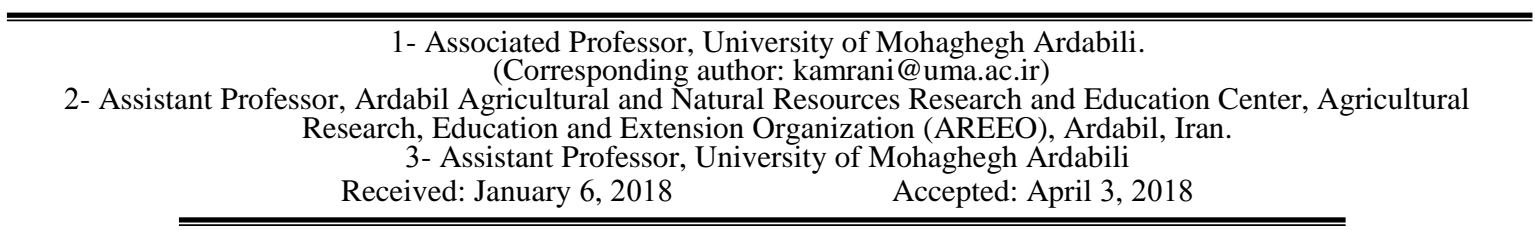

\begin{abstract}
Among different environmental stresses, drought is of great importance that induces a highly negative effect on crop production. In order to evaluate drought tolerance in dryland wheat genotypes, 36 genotypes were studied in a randomized complete block design with three replications under rainfed (drought stress) and supplemental irrigation conditions during 2016-2017 growing season in Research Farm of Moghan College of Agriculture and Natural Resources. Results showed that there are significant differences among genotypes for grain yield in both rainfed and supplemental irrigation conditions. Combined analysis of variance over two experiments showed that drought stress significantly increased grain yield. Under supplemental irrigation conditions, the genotypes 22,34 and 21 had the highest grain yield by an average of $3.143,3.089$ and $2.921 \mathrm{t} / \mathrm{ha}$ and under rainfed condition, genotypes 20, 22 and 27 produced the highest grain yield with an average of 2.647, 2.610 and $2.558 \mathrm{t} / \mathrm{ha}$, respectively. In order to identify drought tolerance genotypes, drought resistant indices such as tolerance index (TOL), stress susceptibility index (SSI), mean productivity (MP), geometric mean productivity (GMP), stress tolerance index (STI) and yield stability index (YSI) were used. GMP, MP and STI indices were highly correlated with grain yield under both stress and non-stress conditions. Therefore, they were introduced as suitable indices to identify superior genotypes for both environmental conditions. Based on principal component analysis and three dimensional graph, genotypes 22, 34, 33 and 15 were identified as drought tolerant genotypes and 7,17,14 and 1 were identified as drought sensitive genotypes. Cluster analysis with Ward's method based on grain yield under supplemental irrigation (Yp) and rainfed (Ys) conditions and drought tolerance indices divided the 36 genotypes into four major groups. Therefore, this result was consistent by results from principal component analysis.
\end{abstract}

Keywords: Bread wheat, Supplemental irrigation, Drought stress, Grain yield, Drought tolerance indices 